\title{
NONCOMMUTATIVE SCHUR FUNCTIONS, SWITCHBOARDS, AND SCHUR POSITIVITY
}

\author{
JONAH BLASIAK AND SERGEY FOMIN
}

\begin{abstract}
We review and further develop a general approach to Schur positivity of symmetric functions based on the machinery of noncommutative Schur functions. This approach unifies ideas of Assaf [1, 3], Lam [22, and Greene and the second author [11].
\end{abstract}

\section{INTRODUCTION}

Schur functions form the most important basis in the ring of symmetric functions, and the problem of expanding various families of symmetric functions with respect to this basis arises in many mathematical contexts. One is particularly interested in obtaining manifestly positive combinatorial descriptions for the coefficients in various Schur expansions, the celebrated Littlewood-Richardson Rule being the prototypical example.

One powerful approach to this class of problems relies on the idea of generalizing the notion of a Schur function to the noncommutative case, or more precisely to noncommutative rings whose generators (the "noncommuting variables") satisfy some carefully chosen relations, making it possible to retain many key features of the classical theory of symmetric functions. The first implementation of this idea goes back to the work of Lascoux and Schützenberger in the 1970's [25, 31]. They showed that the plactic algebra (i.e., the ring defined by the Knuth relations) contains a subalgebra isomorphic to the ring of symmetric functions, and moreover this subalgebra comes naturally equipped with a basis of noncommutative versions of Schur functions. In the 1990's, Greene and the second author [11] generalized this approach by replacing some of the plactic relations by weaker four-term relations. By studying noncommutative versions of Schur functions in the algebra thus defined, they obtained, in a uniform fashion, positive Schur expansions for a large class of (ordinary) symmetric functions that includes the Stanley symmetric functions and stable Grothendieck polynomials. As explained below, the construction in [1] can be further generalized to include such important examples as LLT and Macdonald polynomials.

LLT polynomials are a family of symmetric functions introduced by Lascoux, Leclerc, and Thibon [24]. While LLT polynomials were shown to be Schur positive [15, 27] using

Date: October 1, 2015.

2010 Mathematics Subject Classification. Primary 05E05. Secondary 05A15, 16 S99.

Key words and phrases. Noncommutative Schur function, switchboard, D graph, LLT polynomial, Schur positivity.

The authors were supported by NSF Grants DMS-14071174 (J. B.) and DMS-1361789 (S. F.). 
Kazhdan-Lusztig theory, it remains a fundamental open problem to find an explicit positive combinatorial formula for the coefficients in their Schur expansions. A solution to this problem would also settle the analogous problem for Macdonald polynomials, since the Haglund-Haiman-Loehr formula [16] expresses an arbitrary transformed Macdonald polynomial as a positive sum of LLT polynomials.

We are optimistic that the noncommutative Schur function approach can be successfully implemented to obtain positive combinatorial formulas for the Schur expansions of LLT and Macdonald polynomials. The first step in this direction was made by Lam [22] who used a variation of this approach to produce formulas - combinatorial but not manifestly positive - for the coefficients appearing in the Schur expansions of LLT polynomials. In a separate development, Assaf [1] introduced variants of Knuth equivalence as a combinatorial tool to study the Schur positivity phenomenon for LLT polynomials.

This paper extends the setup of [11] to encompass Lam's work and give an algebraic framework for Assaf's equivalences. Specifically, we

- strengthen the main result of [11] (Theorem 2.14, proof in Section 9);

- introduce combinatorial gadgets called switchboards (Definition 3.1), which were inspired by the D graphs of Assaf [1];

- associate a symmetric function to each switchboard (Definitions 3.4/2.5) and show that the class of symmetric functions so defined includes many important families such as LLT polynomials and all examples from [11] (Sections [3 5);

- give a (counter)example of a switchboard whose symmetric function is not Schur positive (Corollary [3.9);

- recall the main result of [5], a positive combinatorial formula for the Schur expansion of an LLT polynomial indexed by a 3-tuple of skew shapes (Theorem 4.22);

- investigate the phenomenon of monomial positivity of noncommutative Schur functions (see (2.12) ) in various rings (Sections 6 7).

We also obtain a "tightness" result (Theorem 2.10, proof in Section 8) which states that in a given ring satisfying the basic requirements of our setup, the noncommutative Schur functions are monomial positive if and only if the symmetric functions naturally associated to this ring are Schur positive. In other words, the noncommutative Schur function approach gives an equivalent reformulation of the Schur positivity problem.

Section 2 of the paper serves as its "extended abstract."

This paper can be viewed as a "prequel" to (and, partly, a review of) the closely related papers [5, 6] by the first author, and to his paper [4] with R. Liu.

Acknowledgments. We thank Sami Assaf, Anna Blasiak, Thomas Lam, and Bernard Leclerc for helpful discussions, and Elaine So and Xun Zhu for help typing and typesetting figures. This project began while the first author was a postdoc at the University of Michigan. He is grateful to John Stembridge for his generous advice and many detailed discussions. 


\section{Noncommutative Schur functions And Schur Positivity}

Let $\mathcal{U}=\mathbb{Z}\left\langle u_{1}, u_{2}, \ldots, u_{N}\right\rangle$ be the free associative ring with generators $u_{1}, u_{2}, \ldots, u_{N}$, which we regard as "noncommuting variables." For $S \subset\{1, \ldots, N\}$ and $k \in \mathbb{Z}$, we define the noncommutative elementary symmetric function $e_{k}\left(\mathbf{u}_{S}\right)$ by

$$
e_{k}\left(\mathbf{u}_{S}\right)=\sum_{\substack{i_{1}>i_{2}>\cdots>i_{k} \\ i_{1}, \ldots, i_{k} \in S}} u_{i_{1}} u_{i_{2}} \cdots u_{i_{k}}
$$

by convention, $e_{0}\left(\mathbf{u}_{S}\right)=1$ and $e_{k}\left(\mathbf{u}_{S}\right)=0$ for $k<0$ or $k>|S|$ (cardinality of $S$ ). For $S=\{1, \ldots, N\}$, we use the notation $e_{k}(\mathbf{u})$.

Throughout this paper an ideal will always mean a two-sided ideal.

Theorem 2.1. For an ideal $I$ of $\mathcal{U}$, the following are equivalent:

- the ideal I includes the elements

$$
\begin{array}{ll}
u_{b}^{2} u_{a}+u_{a} u_{b} u_{a}-u_{b} u_{a} u_{b}-u_{b} u_{a}^{2} & (a<b), \\
u_{b} u_{c} u_{a}+u_{a} u_{c} u_{b}-u_{b} u_{a} u_{c}-u_{c} u_{a} u_{b} & (a<b<c), \\
u_{c} u_{b} u_{c} u_{a}+u_{b} u_{c} u_{a} u_{c}-u_{c} u_{b} u_{a} u_{c}-u_{b} u_{c}^{2} u_{a} & (a<b<c) ;
\end{array}
$$

- the noncommutative elementary symmetric functions $e_{k}\left(\mathbf{u}_{S}\right)$ and $e_{\ell}\left(\mathbf{u}_{S}\right)$ commute with each other modulo $I$, for any $k, \ell$ and any $S$ :

$$
e_{k}\left(\mathbf{u}_{S}\right) e_{\ell}\left(\mathbf{u}_{S}\right) \equiv e_{\ell}\left(\mathbf{u}_{S}\right) e_{k}\left(\mathbf{u}_{S}\right) \bmod I .
$$

Remark 2.2. Theorem 2.1] is equivalent to a result by A. N. Kirillov [21, Theorem 2.24]. It generalizes similar results obtained in [6, 11, 22, 28. Specifically, [11, Lemma 3.1], [22, Theorem 3], [6, Lemma 2.2], and [28, Theorem 2] assert, in the notation to be introduced below, that the $e_{k}(\mathbf{u})$ commute modulo the ideals $I_{\mathrm{B}}, J_{\mathrm{L}, k}, I_{\mathrm{S}}+I_{\text {st }}$, and the triples ideal with all rotation triples, respectively; cf. Definitions 2.12, 4.6, 2.17/3.14, and 6.3,

Theorem 2.1 has the following elegant reformulation.

Theorem 2.3. The commutation relations (2.5) hold for all $k, \ell$, and $S \subset\{1, \ldots N\}$ provided they hold for $k=1, \ell \in\{2,3\}$, and $|S| \leq 3$.

It is quite miraculous that the special cases $|S| \leq 3$ of the commutation relations (2.5) (i.e., the cases involving two or three variables only) are sufficient to get all of them. An additional miracle is that the relation $e_{2}\left(\mathbf{u}_{S}\right) e_{3}\left(\mathbf{u}_{S}\right) \equiv e_{3}\left(\mathbf{u}_{S}\right) e_{2}\left(\mathbf{u}_{S}\right)$ for $|S|=3$ is not needed: all instances of (2.5), including the latter, are a consequence of $e_{1}\left(\mathbf{u}_{S}\right) e_{2}\left(\mathbf{u}_{S}\right) \equiv$ $e_{2}\left(\mathbf{u}_{S}\right) e_{1}\left(\mathbf{u}_{S}\right)$ and $e_{1}\left(\mathbf{u}_{S}\right) e_{3}\left(\mathbf{u}_{S}\right) \equiv e_{3}\left(\mathbf{u}_{S}\right) e_{1}\left(\mathbf{u}_{S}\right)$ for $|S| \leq 3$.

We denote by $I_{\mathrm{C}}$ the ideal in $\mathcal{U}$ generated by the elements (2.2)-(2.4) in Theorem 2.1 All ideals $I \subset \mathcal{U}$ considered in this paper contain $I_{\mathrm{C}}$.

Let $x$ be a formal variable that commutes with $u_{1}, \ldots, u_{N}$. The elements $e_{k}(\mathbf{u})$ can be packaged into the generating function

$$
E(x)=\sum_{k=0}^{N} x^{k} e_{k}(\mathbf{u})=\left(1+x u_{N}\right) \cdots\left(1+x u_{1}\right) \in \mathcal{U}[x] .
$$


The condition that the $e_{k}(\mathbf{u})$ commute pairwise modulo $I_{\mathrm{C}}$ is equivalent to the generating function congruence $E(x) E(y) \equiv E(y) E(x)$ modulo $I_{\mathrm{C}}$, or more precisely modulo the corresponding ideal $I_{\mathrm{C}}[x, y] \subset \mathcal{U}[x, y]$. Here $y$ is another formal variable that commutes with $x, u_{1}, \ldots, u_{N}$.

The noncommutative complete homogeneous symmetric functions are defined by

$$
h_{\ell}(\mathbf{u})=\sum_{1 \leq i_{1} \leq i_{2} \leq \cdots \leq i_{\ell} \leq N} u_{i_{1}} u_{i_{2}} \cdots u_{i_{\ell}}
$$

for $\ell>0$; by convention, $h_{0}(\mathbf{u})=1$ and $h_{\ell}(\mathbf{u})=0$ for $\ell<0$. Similarly to (2.6), we set

$$
H(x)=\sum_{\ell=0}^{\infty} x^{\ell} h_{\ell}(\mathbf{u})=\left(1-x u_{1}\right)^{-1} \cdots\left(1-x u_{N}\right)^{-1} \in \mathcal{U}[[x]] .
$$

Corollary 2.4. Let $I \subset \mathcal{U}$ be an ideal containing $I_{\mathrm{C}}$. Then the elements $h_{1}(\mathbf{u}), h_{2}(\mathbf{u}), \ldots$ commute pairwise modulo I. Consequently,

$$
H(x) H(y) \equiv H(y) H(x) \bmod I[[x, y]]
$$

where $x$ and $y$ are formal variables commuting with each other and with $u_{1}, \ldots, u_{N}$.

Proof. The identity $H(x) E(-x)=1$ implies that for any $m>0$,

$$
h_{m}(\mathbf{u})-h_{m-1}(\mathbf{u}) e_{1}(\mathbf{u})+h_{m-2}(\mathbf{u}) e_{2}(\mathbf{u})-\cdots+(-1)^{m} e_{m}(\mathbf{u})=0 .
$$

Thus one can recursively express $h_{1}(\mathbf{u}), h_{2}(\mathbf{u}), \ldots$ in terms of $e_{1}(\mathbf{u}), e_{2}(\mathbf{u}), \ldots$, and the claim follows from Theorem 2.1.

We denote by $\mathcal{U}^{*}$ the $\mathbb{Z}$-module freely spanned by the set of words in the alphabet $\{1, \ldots, N\}$. There is a natural pairing $\langle\cdot, \cdot\rangle$ between the spaces $\mathcal{U}$ and $\mathcal{U}^{*}$ in which the basis of noncommutative monomials is dual to the basis of words. More precisely, if $\mathbf{u}_{\mathrm{w}}=u_{\mathrm{w}_{1}} \cdots u_{\mathrm{w}_{n}} \in \mathcal{U}$ is a monomial corresponding to the word $\mathrm{w}=\mathrm{w}_{1} \cdots \mathrm{w}_{n} \in \mathcal{U}^{*}$, and $\mathbf{v} \in \mathcal{U}^{*}$ is another word, then $\left\langle\mathbf{u}_{\mathrm{w}}, \mathbf{v}\right\rangle=\delta_{\mathrm{vw}}$.

For an ideal $I \subset \mathcal{U}$, we denote by $I^{\perp}$ the orthogonal complement

$$
I^{\perp}=\left\{\gamma \in \mathcal{U}^{*} \mid\langle z, \gamma\rangle=0 \text { for all } z \in I\right\} .
$$

Informally speaking, pairing with the elements of $I^{\perp}$ is tantamount to working modulo $I$ : for $f, g \in \mathcal{U}$, the congruence $f \equiv g \bmod I$ implies $\langle f, \gamma\rangle=\langle g, \gamma\rangle$ for all $\gamma \in I^{\perp}$. Put another way, any element of $\mathcal{U} / I$ has a well-defined pairing with any element of $I^{\perp}$.

As shown in [11, noncommutative symmetric functions $e_{k}(\mathbf{u})$ and $h_{\ell}(\mathbf{u})$ can be used to define and study a large class of symmetric functions in the usual sense, i.e., formal power series (in commuting variables) which are symmetric under permutations of these variables. This is done as follows.

Definition 2.5. Let $x_{1}, x_{2}, \ldots$ be formal variables which commute with each other and with $u_{1}, \ldots, u_{N}$. Define the noncommutative Cauchy product $\Omega(\mathbf{x}, \mathbf{u}) \in \mathcal{U}\left[\left[x_{1}, x_{2}, \ldots\right]\right]$ by

$$
\Omega(\mathbf{x}, \mathbf{u})=H\left(x_{1}\right) H\left(x_{2}\right) \cdots=\prod_{j=1}^{\infty} \prod_{i=1}^{N}\left(1-x_{j} u_{i}\right)^{-1}=\prod_{j=1}^{\infty} \sum_{\ell=0}^{\infty} x_{j}^{\ell} h_{\ell}(\mathbf{u})
$$


(cf. (2.7)); here the notation $\prod$ always means that the factors are multiplied left-to-right. Now, for $\gamma \in \mathcal{U}^{*}$, we define the formal power series $F_{\gamma}(\mathbf{x}) \in \mathbb{Z}\left[\left[x_{1}, x_{2}, \ldots\right]\right]$ by

$$
F_{\gamma}(\mathbf{x})=\langle\Omega(\mathbf{x}, \mathbf{u}), \gamma\rangle
$$

where $\langle\cdot, \cdot\rangle$ is the extension of the pairing between $\mathcal{U}$ and $\mathcal{U}^{*}$ to the bilinear pairing between $\mathcal{U}\left[\left[x_{1}, x_{2}, \ldots\right]\right]$ and $\mathcal{U}^{*}$ in which $\left\langle f \mathbf{u}_{\mathrm{w}}, \mathbf{v}\right\rangle=f \delta_{\mathrm{vw}}$ for any $f \in \mathbb{Z}\left[\left[x_{1}, x_{2}, \ldots\right]\right]$.

An alternative definition of $F_{\gamma}(\mathbf{x})$ can be given in terms of quasisymmetric functions. Collecting the terms in (2.9) involving each noncommutative monomial $\mathbf{u}_{\mathrm{w}}$, we obtain

$$
\Omega(\mathbf{x}, \mathbf{u})=\sum_{\mathrm{w}} Q_{\operatorname{Des}(\mathrm{w})}(\mathbf{x}) \mathbf{u}_{\mathrm{w}}
$$

where $\operatorname{Des}(\mathrm{w})=\left\{i \in\{1, \ldots, n-1\} \mid \mathrm{w}_{i}>\mathrm{w}_{i+1}\right\}$ denotes the descent set of a word $\mathrm{w}=\mathrm{w}_{1} \cdots \mathrm{w}_{n}$, and

$$
Q_{\operatorname{Des}(\mathrm{w})}(\mathbf{x})=\sum_{\substack{1 \leq i_{1} \leq \cdots \leq i_{n} \\ j \in \operatorname{Des}(\mathrm{w}) \Longrightarrow i_{j}<i_{j+1}}} x_{i_{1}} \cdots x_{i_{n}}
$$

is the corresponding fundamental quasisymmetric function (I. Gessel [14]). Consequently, for a vector $\gamma=\sum_{w} \gamma_{w} w \in \mathcal{U}^{*}$, we have

$$
F_{\gamma}(\mathbf{x})=\sum_{\mathrm{w}} \gamma_{\mathrm{w}} Q_{\operatorname{Des}(\mathrm{w})}(\mathbf{x})
$$

Thus the map $\gamma \mapsto F_{\gamma}(\mathbf{x})$ is simply the linear map $\mathcal{U}^{*} \rightarrow \mathbb{Z}\left[\left[x_{1}, x_{2}, \ldots\right]\right]$ that sends each word $\mathrm{w}$ to the fundamental quasisymmetric function associated with the descent set of $\mathrm{w}$.

Proposition 2.6. If $\gamma \in I_{\mathrm{C}}^{\perp}$, then $F_{\gamma}(\mathbf{x})$ is symmetric in $x_{1}, x_{2}, \ldots$

Proof. Let $\Omega_{j}(\mathbf{x}, \mathbf{u})=\cdots H\left(x_{j-1}\right) H\left(x_{j+1}\right) H\left(x_{j}\right) H\left(x_{j+2}\right) \cdots$ denote the result of switching $x_{j}$ and $x_{j+1}$ in $\Omega(\mathbf{x}, \mathbf{u})$. By Corollary $2.4, \Omega_{j}(\mathbf{x}, \mathbf{u}) \equiv \Omega(\mathbf{x}, \mathbf{u}) \bmod I_{\mathrm{C}}\left[\left[x_{1}, x_{2}, \ldots\right]\right]$. Hence $\left\langle\Omega_{j}(\mathbf{x}, \mathbf{u}), \gamma\right\rangle=\langle\Omega(\mathbf{x}, \mathbf{u}), \gamma\rangle$, and the claim follows.

As explained in Sections 4 and 5, the class of symmetric functions $F_{\gamma}$ includes several important families such as Stanley symmetric functions and LLT polynomials. The following construction provides a powerful tool for studying the coefficients of the expansions of the symmetric functions $F_{\gamma}$ in the basis of Schur functions.

Definition 2.7 (Noncommutative Schur functions). Let $\lambda=\left(\lambda_{1}, \lambda_{2}, \ldots\right)$ be an integer partition. Let $\lambda^{\prime}$ be the conjugate partition, and let $t=\lambda_{1}$ be the number of parts of $\lambda^{\prime}$. The noncommutative Schur function $\mathfrak{J}_{\lambda}(\mathbf{u}) \in \mathcal{U}$ is given by the following noncommutative version of the classical Kostka-Naegelsbach determinantal formula $s_{\lambda}=\operatorname{det}\left(e_{\lambda_{i}^{\prime}+j-i}\right)$ :

$$
\mathfrak{J}_{\lambda}(\mathbf{u})=\sum_{\pi \in \mathcal{S}_{t}} \operatorname{sgn}(\pi) e_{\lambda_{1}^{\prime}+\pi(1)-1}(\mathbf{u}) e_{\lambda_{2}^{\prime}+\pi(2)-2}(\mathbf{u}) \cdots e_{\lambda_{t}^{\prime}+\pi(t)-t}(\mathbf{u}) .
$$

Remark 2.8. Alternatively, $\mathfrak{J}_{\lambda}(\mathbf{u})$ can be defined by the noncommutative version of the Jacobi-Trudi formula:

$$
\mathfrak{J}_{\lambda}(\mathbf{u})=\sum_{\pi \in \mathcal{S}_{\ell}} \operatorname{sgn}(\pi) h_{\lambda_{1}+\pi(1)-1}(\mathbf{u}) h_{\lambda_{2}+\pi(2)-2}(\mathbf{u}) \cdots h_{\lambda_{\ell}+\pi(\ell)-\ell}(\mathbf{u})
$$


here $\ell=\lambda_{1}^{\prime}$ is the number of parts of $\lambda$. The fact that the right-hand sides of (2.12) and (2.13) are congruent to each other modulo $I_{\mathrm{C}}$ can be established by the argument given in the proof of Proposition 2.9 below.

The closely related noncommutative Schur functions $s_{\lambda}(\mathbf{u})$ introduced and studied in [11] were defined there in terms of semistandard Young tableaux, cf. Theorem 5.1. While $s_{\lambda}(\mathbf{u}) \equiv \mathfrak{J}_{\lambda}(\mathbf{u})$ modulo the ideals considered in [11], the two definitions may diverge for other ideals containing $I_{\mathrm{C}}$.

The next result is a version of a simple, yet important, observation made in [11].

Proposition 2.9. For any $\gamma \in I_{\mathrm{C}}^{\perp}$, we have

$$
F_{\gamma}(\mathbf{x})=\sum_{\lambda} s_{\lambda}(\mathbf{x})\left\langle\mathfrak{J}_{\lambda}(\mathbf{u}), \gamma\right\rangle
$$

Proof. Comparing (2.14) to (2.10), we see that it suffices to show

$$
\Omega(\mathbf{x}, \mathbf{u}) \equiv \sum_{\lambda} s_{\lambda}(\mathbf{x}) \mathfrak{J}_{\lambda}(\mathbf{u}) \bmod I_{\mathrm{C}}[[\mathbf{x}]]
$$

Let $\Lambda(\mathbf{y})$ denote the ordinary ring of symmetric polynomials in $N$ commuting variables $\mathbf{y}=\left(y_{1}, \ldots, y_{N}\right)$. This is a polynomial ring with algebraically independent generators $e_{1}(\mathbf{y}), \ldots, e_{N}(\mathbf{y})$. The ring homomorphism $\psi: \Lambda(\mathbf{y}) \rightarrow \mathcal{U} / I_{\mathrm{C}}$ defined by $e_{k}(\mathbf{y}) \mapsto e_{k}(\mathbf{u})$ sends each Schur polynomial $s_{\lambda}(\mathbf{y})$ to $\mathfrak{J}_{\lambda}(\mathbf{u})$ and sends $h_{m}(\mathbf{y})$ to $h_{m}(\mathbf{u})$. To see the latter, apply $\psi$ to the classical identity $\sum_{\ell+k=m}(-1)^{k} h_{\ell}(\mathbf{y}) e_{k}(\mathbf{y})=0$ and then subtract (2.8) to obtain $\sum_{\ell+k=m}(-1)^{k}\left(\psi\left(h_{\ell}(\mathbf{y})\right)-h_{\ell}(\mathbf{u})\right) e_{k}(\mathbf{u})=0$; the desired fact $\psi\left(h_{m}(\mathbf{y})\right)=h_{m}(\mathbf{u})$ then follows by induction on $m$. Now applying $\psi$ to the classical Cauchy identity

$$
\prod_{j=1}^{\infty} \sum_{\ell=0}^{\infty} x_{j}^{\ell} h_{\ell}(\mathbf{y})=\sum_{\lambda} s_{\lambda}(\mathbf{x}) s_{\lambda}(\mathbf{y})
$$

yields (2.15).

As mentioned earlier, many important families of symmetric functions arise from the above construction. One begins by choosing a particular ideal $I \subset \mathcal{U}$ which contains $I_{\mathrm{C}}$ and is homogeneous with respect to the grading $\operatorname{deg}\left(u_{i}\right)=1$. Typically, one is interested in the subclass of symmetric functions $F_{\gamma}(\mathbf{x})$ labeled by the elements $\gamma \in I^{\perp} \subset I_{\mathrm{C}}^{\perp}$ which are nonnegative integer combinations of words. The goal is to show that any such symmetric function $F_{\gamma}(\mathbf{x})$ is Schur positive, i.e., its expansion in the basis of Schur functions has nonnegative coefficients. An even more ambitious goal is to obtain a manifestly positive combinatorial rule for these coefficients.

Let $\mathcal{U}_{\geq 0} \subset \mathcal{U}$ (resp., $\mathcal{U}_{\geq 0}^{*} \subset \mathcal{U}^{*}$ ) denote the $\mathbb{Z}_{\geq 0}$-cone (that is, the additive monoid) generated by the noncommutative monomials in $\mathcal{U}$ (resp., words in $\mathcal{U}^{*}$ ). To rephrase, these cones consist of all nonnegative integer combinations of monomials (resp., words). The symmetric functions $F_{\gamma}$ of interest to us are labeled by $\gamma \in \mathcal{U}_{\geq 0}^{*} \cap I^{\perp}$.

An element $f \in \mathcal{U}$ is called $\mathbb{Z}$-monomial positive modulo an ideal $I$ if $f \in \mathcal{U}_{\geq 0}+I$, i.e., if $f$ can be written, modulo $I$, as a nonnegative integer combination of noncommutative 
monomials. We say that $f \in \mathcal{U}$ is $\mathbb{Q}$-monomial positive modulo $I$ if some positive integer multiple of $f$ is $\mathbb{Z}$-monomial positive modulo $I$.

Theorem 2.10. For a homogeneous ideal $I \subset \mathcal{U}$ containing $I_{\mathrm{C}}$, the following are equivalent:

(i) all symmetric functions $F_{\gamma}(\mathbf{x})$, for $\gamma \in \mathcal{U}_{\geq 0}^{*} \cap I^{\perp}$, are Schur positive;

(ii) all noncommutative Schur functions $\mathfrak{J}_{\lambda}(\mathbf{u})$ are $\mathbb{Q}$-monomial positive modulo $I$.

In fact, a stronger statement holds: given $I \supset I_{\mathrm{C}}$ and an integer partition $\lambda$, the coefficient of $s_{\lambda}(\mathbf{x})$ in $F_{\gamma}(\mathbf{x})$ is nonnegative for all $\gamma \in \mathcal{U}_{\geq 0}^{*} \cap I^{\perp}$ if and only if $\mathfrak{J}_{\lambda}(\mathbf{u})$ is $\mathbb{Q}$-monomial positive modulo $I$.

Theorem 2.10 can be viewed as a simplified version of [6, Theorem 1.4], which gave a slightly stronger conclusion in a more restricted setting.

Proof of the implication (ii) $\Rightarrow(\mathrm{i})$. By assumption (ii), there is a monomial expansion

$$
c_{\lambda} \mathfrak{J}_{\lambda}(\mathbf{u}) \equiv \sum_{w} a_{\lambda, w} \mathbf{u}_{\mathrm{w}}(\bmod I),
$$

for some positive integer $c_{\lambda}$ and nonnegative integer coefficients $a_{\lambda, w}$. Let

$$
\gamma=\sum_{w} \gamma_{w} w \in I^{\perp}
$$

where all coefficients $\gamma_{w}$ are nonnegative integers. It follows that

$$
c_{\lambda}\left\langle\mathfrak{J}_{\lambda}(\mathbf{u}), \gamma\right\rangle=\left\langle\sum_{w} a_{\lambda, w} \mathbf{u}_{w}, \gamma\right\rangle=\sum_{w} a_{\lambda, w} \gamma_{w} \geq 0 .
$$

It remains to recall that by Proposition 2.9, the coefficient of $s_{\lambda}(\mathbf{x})$ in the Schur expansion of $F_{\gamma}(\mathbf{x})$ is equal to $\left\langle\mathfrak{J}_{\lambda}(\mathbf{u}), \gamma\right\rangle$.

The converse implication (i) $\Rightarrow$ (ii) is proved in Section 8 using Farkas' Lemma from convex analysis. Informally, this implication shows that the noncommutative Schur function approach to proving Schur positivity is a powerful one because it only fails if the symmetric functions to which it is applied are not actually all Schur positive.

Remark 2.11. We note that $\mathbb{Q}$-monomial positivity (2.16) of noncommutative Schur functions $\mathfrak{J}_{\lambda}(\mathbf{u})$ modulo a given ideal $I$ implies more than "just" Schur positivity of the corresponding class of symmetric functions $F_{\gamma}(\mathbf{x})$. It yields a manifestly positive formula for the Schur expansion of $F_{\gamma}(\mathbf{x})$, cf. (2.14) and (2.18):

$$
F_{\gamma}(\mathbf{x})=\sum_{\lambda} s_{\lambda}(\mathbf{x}) c_{\lambda}^{-1} \sum_{w} a_{\lambda, w} \gamma_{w}
$$

If moreover all $\mathfrak{J}_{\lambda}(\mathbf{u})$ are $\mathbb{Z}$-monomial positive modulo $I$, so we can take $c_{\lambda}=1$, then (2.19) becomes a positive combinatorial rule for the Schur expansions of $F_{\gamma}$. (We imagine that a monomial positive expansion for $\mathfrak{J}_{\lambda}(\mathbf{u})$ is established by an explicit formula in which the numbers $a_{\lambda, w}$ count combinatorial objects of some kind.) We suspect that it can happen that $\mathfrak{J}_{\lambda}(\mathbf{u})$ is $\mathbb{Q}$-monomial positive modulo $I$, but not $\mathbb{Z}$-monomial positive modulo $I$. 
Unfortunately, and contrary to what extensive computational experiments initially led us to believe, not all symmetric functions $F_{\gamma}(\mathbf{x})$, for $\gamma \in \mathcal{U}_{\geq 0}^{*} \cap I_{\mathrm{C}}^{\perp}$, are Schur positive. (Equivalently, $\mathbb{Q}$-monomial positivity of $\mathfrak{J}_{\lambda}(\mathbf{u})$ may fail modulo $I_{\mathrm{C}}$.) We present a counterexample in Section 3, cf. Corollary 3.9. This negative result must not however discourage us from pursuing the current line of inquiry: even though Schur positivity may fail in the most general case $I=I_{\mathrm{C}}$, it still holds for many important ideals $I \supset I_{\mathrm{C}}$, or equivalently for the nonnegative vectors $\gamma \in I^{\perp} \subset I_{\mathrm{C}}^{\perp}$.

In each application, the challenge is to find a monomial positive expression for $\mathfrak{J}_{\lambda}(\mathbf{u})$ that holds modulo an appropriately chosen ideal $I$. In doing so, the goal is to choose an ideal $I$ which is "exactly the right size." First of all, $I$ must be small enough so that the symmetric functions of interest to us are of the form $F_{\gamma}$ for $\gamma \in I^{\perp}$. On the other hand, if $I$ is too small, e.g., $I=I_{\mathrm{C}}$, then $\mathfrak{J}_{\lambda}(\mathbf{u})$ is not monomial positive modulo $I$. But there is yet the further subtle consideration: if $I$ is too large, then there are many ways to write $\mathfrak{J}_{\lambda}(\mathbf{u})$ as a positive sum of monomials (modulo $I$ ), making it extremely hard to single out a "canonical one" from this multitude; this is what essentially happens in Lam's approach [22] to LLT polynomials. In conclusion, identifying the "smallest" ideal $I$ such that $\mathfrak{J}_{\lambda}(\mathbf{u})$ is monomial positive modulo $I$ is likely to be of help in finding (and proving) the desired combinatorial rule for such monomial positive expression, and would establish Schur positivity for a wider class of symmetric functions.

Matters are complicated by the fact that the subset of ideals $I \supset I_{\mathrm{C}}$ for which monomial positivity of $\mathfrak{J}_{\lambda}(\mathbf{u})$ holds modulo $I$ does not have a unique minimal element, see Corollary 7.6. Hence it might be too optimistic to hope for an all-encompassing "master Schur positivity theorem," as different symmetric functions $F_{\gamma}$ may be Schur positive for different reasons.

On the bright side, monomial positivity of $\mathfrak{J}_{\lambda}(\mathbf{u})$ can be established for many important ideals $I \supset I_{\mathrm{C}}$, as demonstrated in [11. (The first example of this phenomenon, namely the case of the plactic algebra, goes back to Lascoux and Schützenberger [25, 31].) Our next theorem can be viewed as a strengthening of the main result of [11].

Definition 2.12. Let $I_{\mathrm{B}}$ denote the ideal in $\mathcal{U}$ generated by the elements

$$
\begin{array}{ll}
u_{b}^{2} u_{a}+u_{a} u_{b} u_{a}-u_{b} u_{a} u_{b}-u_{b} u_{a}^{2} & (a<b), \\
u_{b} u_{c} u_{a}-u_{b} u_{a} u_{c} & (a<b<c), \\
u_{a} u_{c} u_{b}-u_{c} u_{a} u_{b} & (a<b<c) .
\end{array}
$$

Lemma 2.13. $I_{\mathrm{B}} \supset I_{\mathrm{C}}$.

Proof. Note that (2.20) coincides with (2.2), while the sum of (2.21) and (2.22) is (2.3). Finally, we get the elements (2.4) as follows: $u_{c} u_{b} u_{c} u_{a}+u_{b} u_{c} u_{a} u_{c}-u_{c} u_{b} u_{a} u_{c}-u_{b} u_{c}^{2} u_{a}=$ $u_{c}\left(u_{b} u_{c} u_{a}-u_{b} u_{a} u_{c}\right)-u_{b}\left(u_{c}^{2} u_{a}+u_{a} u_{c} u_{a}-u_{c} u_{a} u_{c}-u_{c} u_{a}^{2}\right)+\left(u_{b} u_{a} u_{c}-u_{b} u_{c} u_{a}\right) u_{a} \in I_{\mathrm{B}}$.

The ideal $I_{\mathrm{B}}$ is the enlargement of $I_{\mathrm{C}}$ obtained by replacing the generators (2.3) by the "3-letter Knuth elements" (2.21)-(2.22). Curiously, this makes the degree 4 generators (2.4) superfluous, as the above calculation shows. 
Theorem 2.14. The noncommutative Schur functions $\mathfrak{J}_{\lambda}(\mathbf{u})$ are $\mathbb{Z}$-monomial positive modulo $I_{\mathrm{B}}$. Consequently, the symmetric functions $F_{\gamma}(\mathbf{x})$ labeled by $\gamma \in \mathcal{U}_{\geq 0}^{*} \cap I_{\mathrm{B}}^{\perp}$ are Schur positive.

Theorem 2.14 generalizes [11, Lemma 3.2], which used a larger ideal $I_{\bigotimes}$ obtained by adding "Knuth elements" involving two non-adjacent indices, cf. (5.1).

Theorem 2.14 is proved in Section 9, The $\mathbb{Z}$-monomial positive formula that we give for $\mathfrak{J}_{\lambda}(\mathbf{u})$ (modulo $I_{\mathrm{B}}$ ) leads to a manifestly positive combinatorial formula for the Schur expansions of the symmetric functions $F_{\gamma}(\mathbf{x})$ labeled by $\gamma \in \mathcal{U}_{>0}^{*} \cap I_{\mathrm{B}}^{\perp}$, extending the "generalized Littlewood-Richardson rule" of [11, Theorem 1.2] for $\gamma \in \mathcal{U}_{\geq 0}^{*} \cap I_{Q}^{\perp}$. As shown in [11], the latter subclass of $F_{\gamma}$ 's includes Stanley symmetric functions and, more generally, homogeneous components of stable Grothendieck polynomials.

Definition 2.15. For $k$ a positive integer, let $I_{\mathrm{R}, k}$ denote the ideal in $\mathcal{U}$ generated by

$$
\begin{array}{ll}
u_{a}^{2} & \text { for all } a, \\
u_{a} u_{b} u_{a} & \text { for all } a \text { and } b, \\
u_{b} u_{a} u_{c}-u_{b} u_{c} u_{a} & \text { for } c-a>k \text { and } a<b<c, \\
u_{a} u_{c} u_{b}-u_{c} u_{a} u_{b} & \text { for } c-a>k \text { and } a<b<c, \\
u_{b} u_{a} u_{c}-u_{a} u_{c} u_{b} & \text { for } c-a \leq k \text { and } a<b<c, \\
u_{b} u_{c} u_{a}-u_{c} u_{a} u_{b} & \text { for } c-a \leq k \text { and } a<b<c .
\end{array}
$$

It is straightforward to verify that $I_{\mathrm{R}, k} \supset I_{\mathrm{C}}$.

In Section 4, by recasting the work of Assaf [1, 3] through the perspective outlined in this paper, we explain that the class of symmetric functions $F_{\gamma}$ labeled by nonnegative vectors $\gamma \in I_{\mathrm{R}, k}^{\perp}$ includes the LLT polynomials of Lascoux, Leclerc, and Thibon [24]; more precisely, it includes the coefficients of powers of $q$ in LLT polynomials indexed by an arbitrary $k$-tuple of skew shapes. Furthermore this class of symmetric functions $F_{\gamma}$ includes the (coefficients of $q^{i} t^{j}$ in) transformed Macdonald polynomials (cf. [13], [18, Definition 3.5.2]); this is because Haglund, Haiman, and Loehr [16] showed that any transformed Macdonald polynomial is a nonnegative integer combination of LLT polynomials. (Recall that the map $\gamma \mapsto F_{\gamma}$ is linear.)

The following statement is inspired by (and is implicit in) the work of Assaf.

Conjecture 2.16. For any integer partition $\lambda$ and any positive integer $k$, the noncommutative Schur function $\mathfrak{J}_{\lambda}(\mathbf{u})$ is $\mathbb{Z}$-monomial positive modulo $I_{\mathrm{R}, k}$. Consequently, the symmetric functions $F_{\gamma}(\mathbf{x})$ labeled by $\gamma \in \mathcal{U}_{\geq 0}^{*} \cap I_{\mathrm{R}, k}$ are Schur positive.

As explained above, the statement in Conjecture 2.16 is stronger than Schur positivity of LLT and Macdonald polynomials. More importantly (cf. Remark 2.11), an explicit formula expressing $\mathfrak{J}_{\lambda}(\mathbf{u})$ as a sum of noncommutative monomials modulo $I_{\mathrm{R}, k}$ would immediately yield a Littlewood-Richardson-type rule for LLT and Macdonald polynomials.

Thus far, the main new result arising from the noncommutative Schur function approach to LLT positivity is an explicit $\mathbb{Z}$-monomial positive expression for $\mathfrak{J}_{\lambda}(\mathbf{u})$ modulo 
$I_{\mathrm{L}, 3}$ which was obtained in [5]. (Here $I_{\mathrm{L}, k} \supset I_{\mathrm{R}, k}$ is the ideal associated with Lam's algebra of ribbon Schur operators, cf. 22. and Definition 4.6.) This result yields a positive combinatorial formula for the Schur expansion of LLT polynomials indexed by 3-tuples of skew shapes and for the Schur expansion of transformed Macdonald polynomials indexed by shapes with 3 columns; see Theorem 4.22 and (4.17).

As the above discussions indicate, our efforts to create a general framework for the study of Schur positivity of symmetric functions $F_{\gamma}$ have been driven by potential applications. Since these symmetric functions are labeled by nonnegative vectors $\gamma$ chosen from the orthogonal complement $I^{\perp}$ of some ideal $I \supset I_{\mathrm{C}}$, our goal is to establish Schur positivity for $I^{\perp}$ as large as possible, or equivalently for $I$ as small as possible.

It turns out that all ideals $I \supset I_{\mathrm{C}}$ arising in important applications known to us contain the following ideal. (The choice of its name will become clear soon.)

Definition 2.17. The switchboard ideal $I_{\mathrm{S}}$ is the ideal in $\mathcal{U}$ generated by the elements

$$
\begin{array}{lc}
u_{b}^{2} u_{a}+u_{a} u_{b} u_{a}-u_{b} u_{a} u_{b}-u_{b} u_{a}^{2} & (b-a=1), \\
u_{b}^{2} u_{a}-u_{b} u_{a} u_{b} & (b-a \geq 2), \\
u_{b} u_{a}^{2}-u_{a} u_{b} u_{a} & (b-a \geq 2), \\
u_{b} u_{c} u_{a}+u_{a} u_{c} u_{b}-u_{b} u_{a} u_{c}-u_{c} u_{a} u_{b} & (a<b<c) .
\end{array}
$$

Lemma 2.18. $I_{\mathrm{S}} \supset I_{\mathrm{C}}$.

Proof. The span of (2.29)-(2.31) contains (2.2), while (2.32) is the same as (2.3). Finally, the elements (2.4) are obtained as follows: $u_{c} u_{b} u_{c} u_{a}+u_{b} u_{c} u_{a} u_{c}-u_{c} u_{b} u_{a} u_{c}-u_{b} u_{c}^{2} u_{a}=$ $u_{b}\left(u_{c} u_{a} u_{c}-u_{c}^{2} u_{a}\right)+u_{c}\left(u_{b} u_{c} u_{a}+u_{a} u_{c} u_{b}-u_{b} u_{a} u_{c}-u_{c} u_{a} u_{b}\right)+\left(u_{c}^{2} u_{a}-u_{c} u_{a} u_{c}\right) u_{b} \in I_{\mathrm{S}}$.

Remark 2.19. We believe that the ideals $I_{\mathrm{B}}$ and $I_{\mathrm{S}}$ are the smallest "natural" ideals containing $I_{\mathrm{C}}$ which are generated in degrees $\leq 3$. (There is a precise statement along these lines, which we omit.) Note that among the generators of $I_{\mathrm{C}}$, the only ones of degree $>3$ are those listed in (2.4).

The inclusions between the main ideals studied in this paper are summarized in Figure1 (several of these ideals are yet to be defined). Except for $I_{\mathrm{B}}$ and $I_{\mathrm{C}}$, all these ideals contain the switchboard ideal $I_{\mathrm{S}}$. Our main focus henceforth will be on the study of the latter.

$$
\begin{array}{|ccccccccc|}
\hline I_{\mathrm{C}} & \subset & I_{\mathrm{S}} & \subset & I_{\mathrm{R}, k} & \subset & I_{\mathrm{A}, k} & \subset & I_{\mathrm{L}, k} \\
\cap & & \cap & & & & & & \\
I_{\mathrm{B}} & \subset & I_{Q} & \subset & I_{\text {plac }} & & & & \\
& & \cap & & & & & & \\
& & I_{\mathrm{H}} & \subset & I_{\text {nplac }} & \subset & I_{\text {nCox }} & & \\
\hline
\end{array}
$$

Figure 1: The main ideals studied in this paper. All of them contain $I_{\mathrm{C}}$. Schur positivity of the symmetric functions $F_{\gamma}$, for $\gamma \in \mathcal{U}_{\geq 0}^{*} \cap I^{\perp}$, is known to hold for $I \supset I_{\mathrm{B}}$ and $I=I_{\mathrm{L}, k}$; is known to fail for $I \subset I_{\mathrm{S}}$; and is conjectured for $I_{\mathrm{R}, k}$ and $I_{\mathrm{A}, k}$. 


\section{Switchboards}

In this section, we introduce a class of labeled graphs called switchboards. They serve as a combinatorial tool for studying the symmetric functions $F_{\gamma}$ associated to the switchboard ideal $I_{\mathrm{S}}$, that is, those labeled by vectors $\gamma \in \mathcal{U}_{\geq 0}^{*} \cap I_{\mathrm{S}}^{\perp}$. This notion is crucial for unifying the perspectives of [1], [11], and [22].

Definition 3.1 (Switchboards). Let $\mathrm{w}=\mathrm{w}_{1} \cdots \mathrm{w}_{n}$ and $\mathrm{w}^{\prime}=\mathrm{w}_{1}^{\prime} \cdots \mathrm{w}_{n}^{\prime}$ be two words of the same length $n$ in the alphabet $\{1, \ldots, N\}$. We say that $\mathrm{w}$ and $\mathrm{w}^{\prime}$ are related by a switch in position $i$ if $\mathrm{w}_{j}=\mathrm{w}_{j}^{\prime}$ for $j \notin\{i-1, i, i+1\}$, while the unordered pair $\left\{\mathrm{w}_{\mathrm{i}-1} \mathrm{w}_{\mathrm{i}} \mathrm{w}_{\mathrm{i}+1}, \mathrm{w}_{i-1}^{\prime} \mathrm{w}_{i}^{\prime} \mathrm{w}_{i+1}^{\prime}\right\}$ fits one of the following patterns (cf. Figure 2):

- $\{$ bac, bca $\}$ or $\{$ acb, cab $\}$, with $a<b<c$ (a Knuth switch);

- $\{\mathrm{bac}, \mathrm{acb}\}$ or $\{\mathrm{bca}, \mathrm{cab}\}$, with $a<b<c$ (a rotation switch);

- $\{$ bab, bba $\}$ or $\{$ aba, baa $\}$, with $a<b$ (a Knuth switch);

- $\{$ bab, aba $\}$ or $\{$ bba, baa $\}$, with $b=a+1$ (a braid/idempotent switch).

A switchboard is an edge-labeled graph $\Gamma$ on a vertex set of words of fixed length $n$ in the alphabet $\{1, \ldots, N\}$ with edge labels from the set $\{2,3, \ldots, n-1\}$ such that each $i$-edge (i.e., an edge labeled $i$ ) corresponds to a switch in position $i$, and each vertex in $\Gamma$ which has exactly one descent in positions $i-1$ and $i$ belongs to exactly one $i$-edge.

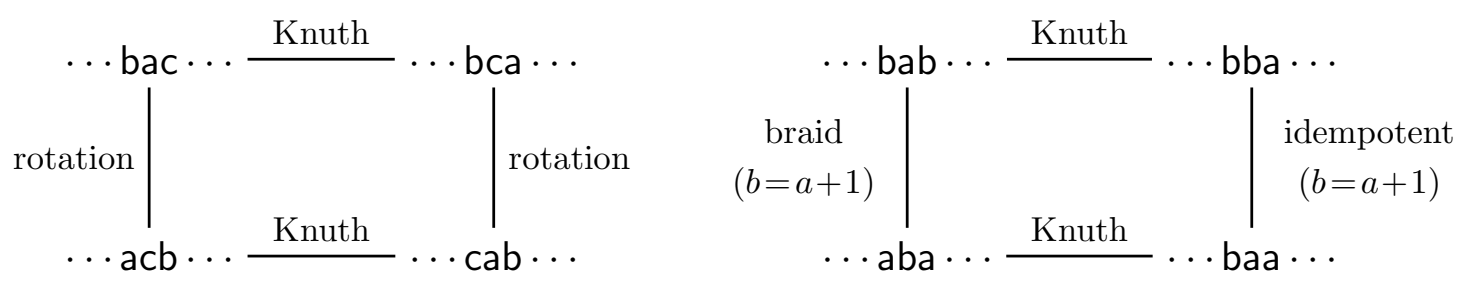

Figure 2: Switches of different types.

Examples of switchboards can be found in Figures 3-10, In all these figures, we label the Knuth $i$-edges by $i$, and the non-Knuth $i$-edges by $\tilde{i}$.

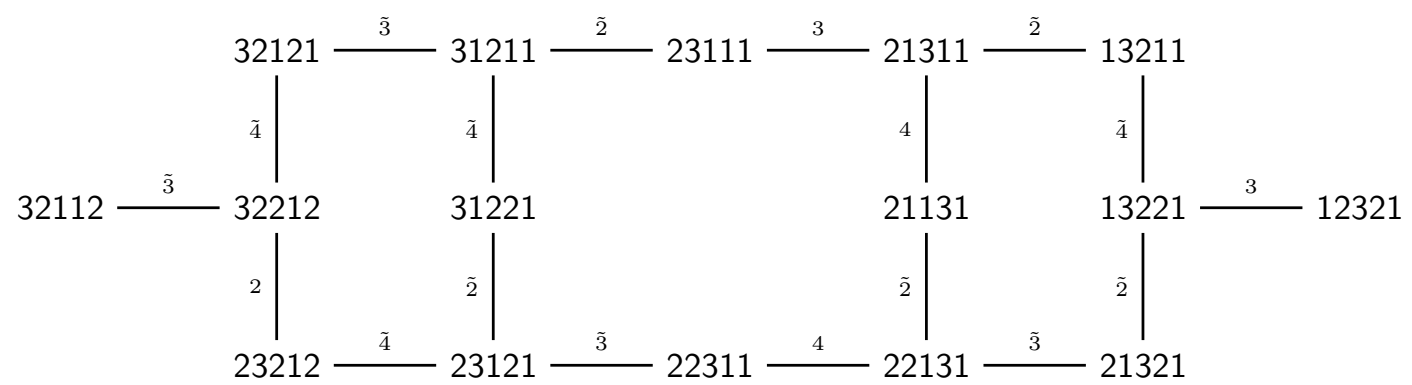

Figure 3: A switchboard with $N=3$ and $n=5$.

Remark 3.2. The notion of a switchboard was inspired by the D graphs of Assaf [1, 2, 3]. More specifically, switchboards are a generalization of the $\mathrm{D}$ graphs $\mathcal{G}_{c, D}^{(k)}$ of [1, $\left.\S 4.2\right]$, which Assaf introduced to study LLT polynomials; cf. Definition 4.12. Switchboards also generalize the $\mathrm{D}_{0}$ graphs of [6], which were in turn motivated by [1, 2, 3]. 
The definition of a switchboard is justified by the following statement, whose verification is straightforward (cf. the proof of [6, Proposition-Definition 3.2]).

Proposition 3.3. Let $W$ be a set of words in $\mathcal{U}^{*}$ of the same length. Then $\sum_{\mathrm{w} \in W} \mathrm{w} \in I_{\mathrm{S}}^{\perp}$ if and only if $W$ is the vertex set of a switchboard.

Definition 3.4. For a switchboard $\Gamma$ with vertex set $W$, we set $F_{\Gamma}(\mathbf{x})=F_{\gamma}(\mathbf{x})$ where $\gamma=\sum_{\mathrm{w} \in W} \mathrm{w}$. Thus $F_{\Gamma}(\mathbf{x})$ is a symmetric function in the variables $\mathbf{x}=\left(x_{1}, x_{2}, \ldots\right)$ defined as the sum of fundamental quasisymmetric functions associated with descent sets of the vertices of $\Gamma$.

Example 3.5. For the switchboard $\Gamma$ in Figure 3 , formula (2.11) gives

$$
\begin{aligned}
F_{\Gamma} & =Q_{2}+Q_{3}+Q_{12}+3 Q_{13}+2 Q_{14}+2 Q_{23}+3 Q_{24}+Q_{34}+Q_{124}+Q_{134} \\
& =s_{32}+s_{311}+s_{221} .
\end{aligned}
$$

The rest of this paper is mostly concerned with the study of switchboards and the associated symmetric functions $F_{\Gamma}$. Comparing Proposition 3.3 to Definitions 2.5 and 3.4 , we see that symmetric functions $F_{\Gamma}$ associated to switchboards are precisely the $F_{\gamma}$ 's labeled by $(0,1)$-vectors $\gamma \in I_{\mathrm{S}}^{\perp}$. (A $(0,1)$-vector is simply a sum of a subset of words in $\mathcal{U}^{*}$.) Restricting the treatment to $(0,1)$-vectors is not much of an imposition since in all important applications the labeling vectors $\gamma$ have this form.

One advantage of switchboards is that one can consider their connected components. This notion involves the edges of a switchboard, not just its vertices, and thus has no direct counterpart for the integer vectors $\gamma$. The following statement is easy to check.

Proposition 3.6. Connected components of a switchboard are switchboards. If two switchboards have disjoint vertex sets consisting of words of the same length, then their union is a switchboard.

Example 3.7. Figure 4 shows two different switchboards $\Gamma$ and $\Gamma^{\prime}$ (one disconnected, the other connected) which have the same set of vertices $W$; consequently $F_{\Gamma}=F_{\Gamma^{\prime}}$. This is an instance of a general phenomenon: if $W$ contains four words of the form shown in Figure 2 on the left, then one can choose either the two Knuth edges or the two rotation edges. A similar phenomenon occurs with 4-tuples of the form shown in Figure 2 on the right (for $b=a+1)$.
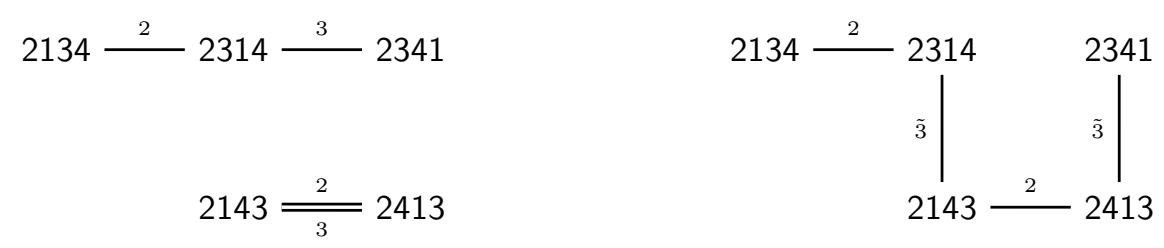

Figure 4: Different switchboards on the same set of vertices, with $N=n=4$. The switchboard $\Gamma$ on the left has two connected components $\Gamma_{\text {top }}$ and $\Gamma_{\text {bottom }}$, with $F_{\Gamma_{\text {top }}}=s_{31}$ and $F_{\Gamma_{\text {bottom }}}=s_{22}$. The switchboard $\Gamma^{\prime}$ on the right is connected, with $F_{\Gamma^{\prime}}=F_{\Gamma}=s_{31}+s_{22}$. 
The results of [1, 5, 11], when translated into the language of switchboards, show that in many important cases, the symmetric functions $F_{\Gamma}(\mathbf{x})$ associated with switchboards $\Gamma$ are Schur positive. Computer experiments supply an extensive list of additional instances of Schur positivity. Unfortunately, there exist switchboards whose symmetric functions are not Schur positive. They are not easy to find, but once a counterexample has been discovered, its verification is a straightforward calculation.

Proposition 3.8. The symmetric function $F_{\Gamma}$ associated with the switchboard shown in Figure可 is not Schur positive: $F_{\Gamma}(\mathbf{x})=s_{321}(\mathbf{x})+s_{2211}(\mathbf{x})-s_{222}(\mathbf{x})$.

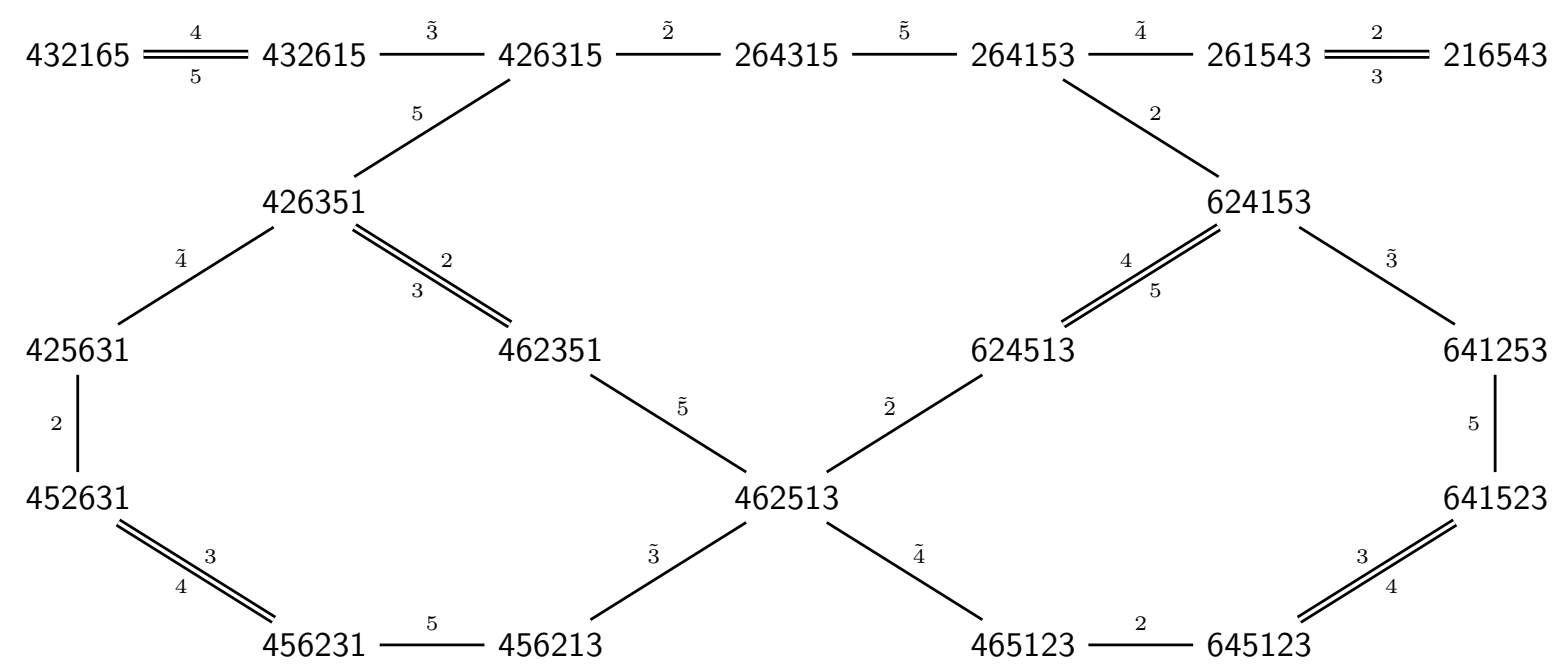

Figure 5: Switchboard $\Gamma$ with $N=n=6, F_{\Gamma}=s_{321}+s_{2211}-s_{222}$.

Proposition 3.8 leads to the following counterexamples.

Corollary 3.9. Conditions (i)-(ii) in Theorem 2.10 fail for $I=I_{\mathrm{S}}$ (hence for $I=I_{\mathrm{C}}$ ), for any $N \geq 6$. Specifically:

(i) the nonnegative vector

$$
\begin{gathered}
\gamma=432165+432615+426315+264315+264153+261543+216543 \\
+426351+624153+425631+462351+624513+641253 \\
+452631+462513+641523+456231+456213+465123+645123
\end{gathered}
$$

lies in $I_{\mathrm{S}}^{\perp}$ but the symmetric function $F_{\gamma}=s_{321}+s_{2211}-s_{222}$ is not Schur positive; (ii) for $\lambda=(2,2,2)$, the noncommutative Schur function $\mathfrak{J}_{\lambda}(\mathbf{u})$ is not $\mathbb{Q}$-monomial positive modulo $I_{\mathrm{S}}$, as witnessed by the fact that the coefficient of $s_{\lambda}$ in $F_{\gamma}$ is -1 for the vector $\gamma \in \mathcal{U}_{\geq 0}^{*} \cap I_{\mathrm{S}}^{\perp}$ defined by (3.1).

Proof. The vector $\gamma$ given in (3.1) is the sum of vertices of the switchboard $\Gamma$ in Figure 5 , Propositions 3.3 and 3.8 imply that $\gamma \in I_{\mathrm{S}}^{\perp}$ and $F_{\gamma}=F_{\Gamma}=s_{321}+s_{2211}-s_{222}$. Theorem 2.10 then yields (ii). 
Given that Schur positivity of $F_{\Gamma}$ fails for general switchboards $\Gamma$, one wonders which additional restrictions imposed on $\Gamma$ would ensure that $F_{\Gamma}$ is Schur positive. One way to obtain results of this kind is to take an ideal $I \supset I_{\mathrm{S}}$ such that $\mathfrak{J}_{\lambda}(\mathbf{u})$ is known to be $\mathbb{Q}$-monomial positive modulo $I$, and consider the corresponding class of switchboards. Various instances of this approach are discussed in subsequent sections of this paper.

An alternative strategy is to examine the counterexamples of switchboards for which Schur positivity fails, recognize their distinctive combinatorial features, and impose restrictions which would rule them out. The following remarks illustrate this approach.

Remark 3.10. A natural subclass of switchboards is defined by the property that "nonoverlapping switches commute:"

if a switchboard has an $i$-edge $\{\mathrm{v}, \mathrm{w}\}$ and a $j$-edge $\left\{\mathrm{v}, \mathrm{w}^{\prime}\right\}$, with $|i-j| \geq 3$, then

it must contain a vertex $\mathrm{v}^{\prime}$ such that $\left\{\mathrm{w}, \mathrm{v}^{\prime}\right\}$ is a $j$-edge and $\left\{\mathrm{w}^{\prime}, \mathrm{v}^{\prime}\right\}$ is an $i$-edge.

(This is the same as D graph axiom 5 from [1].) The switchboard in Figure 5 does not satisfy condition (3.2): starting at $v=426315$ and following the 2-edge and then the 5-edge, we arrive at a different vertex $v s$. following the 5-edge and then the 2-edge. It is natural to ask whether all switchboards satisfying condition (3.2) have Schur positive symmetric functions. Unfortunately this is also false by the example from [6, Theorem 1.5].

Remark 3.11. Another natural restriction is the following "locality" property:

if two words $v$ and $w$ appearing in the same switchboard coincide in positions $(i-1, i, i+1)$, then the $i$-edges incident to $\mathrm{v}$ and $\mathrm{w}$ (if present) are of the same type (i.e., simultaneously Knuth or non-Knuth).

It is easy to see that (3.3) implies (3.2). Hence the switchboard in Figure 5 must violate (3.3). (To see this directly, take $i=5, \mathrm{v}=264315$, and $\mathrm{w}=426315$.) A further strengthening of (3.3) is the following "strong locality" property:

for each $i$, all $i$-edges are of the same type, i.e., all Knuth or all non-Knuth.

Problem 3.12. Is it true that for any switchboard $\Gamma$ satisfying condition (3.3) (resp., the stronger condition (3.4)), the symmetric function $F_{\Gamma}$ is Schur positive?

Definition 3.13. A $\mathrm{D}_{0}$ graph is a switchboard whose words have no repeated letters.

$\mathrm{D}_{0}$ graphs were the main objects of study in [6], and were studied, with slightly different conventions, in [2, 3]. Since switchboards arising in many applications are $\mathrm{D}_{0}$ graphs, it is tempting to speculate that passing from general switchboards in a certain subclass to $\mathrm{D}_{0}$ graphs might help establish Schur positivity. Our investigations show that typically this is not the case. For example, the switchboard $\Gamma$ in Figure 5 is a $\mathrm{D}_{0}$ graph, yet $F_{\Gamma}$ is not Schur positive. The existence of $\mathrm{D}_{0}$ graphs for which Schur positivity fails was already shown in [6], but the example given here is smaller (20 vertices only).

Definition 3.14. Let $I_{\text {st }}$ denote the ideal in $\mathcal{U}$ generated by the monomials $\mathbf{u}_{\mathrm{w}}$ whose associated word $\mathrm{w}$ has a repeated letter.

Roughly speaking, passing from switchboards to $D_{0}$ graphs corresponds to passing from an ideal $I$ to $I+I_{\text {st }}$. As noted above, this enlargement rarely affects Schur positivity. 


\section{LLT POLYNOMIALS}

LLT polynomials are certain $q$-analogs of products of skew Schur functions introduced by Lascoux, Leclerc, and Thibon [24]. In this section, by recasting the work of Lam [22] and Assaf [1, we define a class of switchboards whose symmetric functions are the coefficients of powers of $q$ in an LLT polynomial. We explain how one of the ideas of 1 can be formulated as a conjectural strengthening of LLT positivity (Conjecture 4.21). We conclude by reviewing related work done in [5].

There are two versions of LLT polynomials, which we distinguish following the notation of [15]: the combinatorial LLT polynomials of [24] defined using spin, and the new variant combinatorial LLT polynomials of [17] defined using inversion numbers. Although the theory of noncommutative Schur functions is well suited to studying the former (see [22]), we prefer to work with the latter, in order to be consistent with [1, 5], and also because inversion numbers are easier to calculate than spin.

We begin by recalling from 1 a formula defining LLT polynomials via expansions in fundamental quasisymmetric functions. This will require some preparation.

We adopt the English (matrix-style) convention for drawing skew shapes (=skew Young diagrams) and tableaux, so that row (resp., column) labels start with 1 and increase from north to south (resp., from west to east). For a cell $z$ located in row $a$ and column $b$ in a skew shape $\beta$, the content $c(z)$ of $z$ is defined by $c(z)=b-a$. Here we view $\beta$ as a subset of $\mathbb{Z}_{\geq 1} \times \mathbb{Z}_{\geq 1}$, i.e., we do distinguish between skew shapes that differ by translations.

Throughout this section, $k$ denotes a positive integer. Let

$$
\boldsymbol{\beta}=\left(\beta^{(0)}, \ldots, \beta^{(k-1)}\right)
$$

be a $k$-tuple of skew shapes. The shifted content $\tilde{c}(z)=\tilde{c}_{\boldsymbol{\beta}}(z)$ of a cell $z \in \beta^{(r)}$ is defined by

$$
\tilde{c}(z)=c(z) k+r .
$$

Example 4.1. Let $k=3$ and $\boldsymbol{\beta}=(\square, \boxplus, \boxplus)=(2,33,33) /(1,11,21)$. The only cell $z \in \beta^{(0)}$ is located in row 1 and column 2 , so $c(z)=1$ and $\tilde{c}(z)=3$. The shifted contents of the cells in $\boldsymbol{\beta}$ are shown below:

$$
\left(\begin{array}{ll|l|l|}
\hline 3 & 4 & 7 \\
\hline 1 & 4 \\
\hline
\end{array}, \begin{array}{|l|l}
\hline 2 \\
\hline
\end{array}\right) .
$$

Let $\mathbb{Y}$ denote the set of all Young diagrams. Define a right action of $\mathcal{U}$ on $\mathbb{Z} \mathbb{Y}$ (the free $\mathbb{Z}$-module with basis $\mathbb{Y}$ ) by

$$
\nu \cdot u_{c}= \begin{cases}\mu & \text { if } \mu / \nu \text { is a cell of content } c \\ 0 & \text { otherwise }\end{cases}
$$

Next define a right action of $\mathcal{U}$ on $\mathbb{Z} \mathbb{Y}^{k}$ as follows: for $\boldsymbol{\nu}=\left(\nu^{(0)}, \ldots, \nu^{(k-1)}\right) \in \mathbb{Y}^{k}$, set

$$
\boldsymbol{\nu} \cdot u_{c k+r}=\left(\ldots, \nu^{(r-1)}, \nu^{(r)} \cdot u_{c}, \nu^{(r+1)}, \ldots\right),
$$

for $c \in \mathbb{Z}$ and $r \in\{0,1, \ldots, k-1\}$. Now, for a $k$-tuple $\boldsymbol{\beta}=\boldsymbol{\mu} / \boldsymbol{\nu}$ of skew shapes, set

$$
\mathrm{W}_{k}^{\prime}(\boldsymbol{\beta})=\left\{\mathrm{v} \text { a word in } \mathcal{U}^{*} \mid \boldsymbol{\nu} \cdot \mathbf{u}_{\mathrm{v}}=\boldsymbol{\mu}\right\} .
$$

It is not hard to see that every word in $\mathrm{W}_{k}^{\prime}(\boldsymbol{\beta})$ is a rearrangement of the shifted contents of the cells in $\boldsymbol{\beta}$. 
The last ingredient we shall need is the $k$-inversion statistic $\operatorname{inv}_{k}^{\prime}$. This is the function on words $v=v_{1} v_{2} \cdots \in \mathcal{U}^{*}$ defined as the cardinality of the set of inversions in $v$ formed by pairs of entries which differ by less than $k$ :

$$
\operatorname{inv}_{k}^{\prime}(\mathrm{v})=\mid\left\{(i, j) \mid i<j \text { and } 0<\mathrm{v}_{\mathrm{i}}-\mathrm{v}_{\mathrm{j}}<k\right\} \mid .
$$

Example 4.2. We continue with Example 4.1. Some elements of $W_{3}^{\prime}(\boldsymbol{\beta})$, together with their 3-inversion numbers, are:

\begin{tabular}{l|ccccc}
$\mathrm{v}$ & 42173845 & 34174285 & 83412745 & 48714235 & 28534174 \\
$\operatorname{inv}_{3}^{\prime}(\mathrm{v})$ & 4 & 5 & 5 & 6 & 6
\end{tabular}

Definition 4.3. Let $q$ be a formal parameter. Let $\boldsymbol{\beta}$ be a $k$-tuple of skew shapes in which the shifted contents of all cells lie in $\{1, \ldots, N\}$. The new variant combinatorial LLT polynomial indexed by $\boldsymbol{\beta}$ is defined by

$$
\mathcal{G}_{\boldsymbol{\beta}}(\mathbf{x} ; q)=\sum_{\mathrm{v} \in \mathrm{W}_{k}^{\prime}(\boldsymbol{\beta})} q^{\mathrm{inv}_{k}^{\prime}(\mathrm{v})} Q_{\operatorname{Des}(\mathrm{v})}(\mathbf{x}) .
$$

(We note that no generality is lost by the restriction on the shifted contents of $\boldsymbol{\beta}$ because $\mathcal{G}_{\boldsymbol{\beta}}(\mathbf{x} ; q)$ is unchanged by translating all the $\beta^{(i)}$ horizontally by the same constant.)

Remark 4.4. This family of polynomials was originally defined in [17] as a sum over $k$-tuples of semistandard tableaux with an inversion statistic. An expression in terms of quasisymmetric functions was given by Assaf [1, Corollary 4.3]. Formula (4.3), which appeared in [5, Proposition 2.8], is an adaptation of Assaf's description. Be aware that the words $v$ used herein are inverses of those used in [1]; see [5, §2.5-2.6] for details.

Remark 4.5. The combinatorial LLT polynomials $G_{\mu / \nu}^{(k)}(\mathbf{x} ; \hat{q})$ are defined as generating functions for $k$-ribbon tableaux weighted by spin. (To be precise, [15, 22, 27] use spin whereas [24] uses cospin.) By a result of [17], setting $q=\hat{q}^{-2}$ identifies the two types of LLT polynomials up to a power of $\hat{q}$, once the indexing shapes $\mu / \nu$ and $\boldsymbol{\beta}$ are related via the $k$-quotient correspondence. See [15, Proposition 6.17] for details.

The main open problem in this subfield of algebraic combinatorics concerns the coefficients $\mathfrak{c}_{\boldsymbol{\beta}}^{\lambda}(q)$ appearing in the Schur expansions of LLT polynomials:

$$
\mathcal{G}_{\boldsymbol{\beta}}(\mathbf{x} ; q)=\sum_{\lambda} \mathfrak{c}_{\boldsymbol{\beta}}^{\lambda}(q) s_{\lambda}(\mathbf{x})
$$

Each $\mathfrak{c}_{\boldsymbol{\beta}}^{\lambda}(q)$ is known to be a polynomial in $q$ with nonnegative integer coefficients. In the case that $\boldsymbol{\beta}$ is a tuple of partition shapes, this was established by Leclerc-Thibon [27] and Kashiwara-Tanisaki [19]; the former showed that the coefficients $\mathfrak{c}_{\boldsymbol{\beta}}^{\lambda}(q)$ are essentially parabolic Kazhdan-Lusztig polynomials, and the latter proved geometrically that these polynomials have nonnegative integer coefficients. The general case was established by Grojnowski and Haiman [15], also using Kazhdan-Lusztig theory. Unfortunately, none of the existing approaches produces an explicit positive combinatorial interpretation of $\mathfrak{c}_{\boldsymbol{\beta}}^{\lambda}(q)$, not even a conjectural one. (The approach of [1], though combinatorial, hinges on an intricate algorithm for transforming a D graph into a dual equivalence graph, and has yet to produce explicit formulas for $k>2$.) 
The problem of finding a Littlewood-Richardson-type rule for LLT polynomials is particularly important because its solution would immediately yield a similar rule for transformed Macdonald polynomials: as mentioned earlier, the Haglund-Haiman-Loehr formula [16] expresses the transformed Macdonald polynomials $\tilde{H}_{\mu}(\mathbf{x} ; q, t)$ as positive sums of LLT polynomials.

In [22], Lam introduced his algebra of ribbon Schur operators, providing an elegant algebraic framework for LLT polynomials. This set the stage for applying the theory of noncommutative Schur functions [11] to the study of Schur expansions of LLT polynomials. Following [5], we work with the following slight variant of Lam's construction.

Definition 4.6. Let $\mathcal{U}_{q}=\mathbb{Q}(q) \otimes_{\mathbb{Z}} \mathcal{U}$ where $\mathbb{Q}(q)$ is the field of rational functions in one variable $q$. Lam's ideal $J_{\mathrm{L}, k}$ is the ideal in $\mathcal{U}_{q}$ generated by the elements

$$
\begin{array}{ll}
u_{a}^{2} & \text { for all } a, \\
u_{a+k} u_{a} u_{a+k} & \text { for all } a, \\
u_{a} u_{a+k} u_{a} & \text { for all } a, \\
u_{a} u_{b}-u_{b} u_{a} & \text { for } b-a>k, \\
u_{a} u_{b}-q^{-1} u_{b} u_{a} & \text { for } 0<b-a<k .
\end{array}
$$

We denote $I_{\mathrm{L}, k}=J_{\mathrm{L}, k} \cap \mathcal{U}$ (viewing $\mathcal{U}$ as the $\mathbb{Z}$-subalgebra of $\mathcal{U}_{q}$ generated by 1 and the $u_{i}$ ). In what follows, we mostly work with $J_{\mathrm{L}, k}$ rather than $I_{\mathrm{L}, k}$. Even though it is the latter that is contained in $\mathcal{U}$, we feel that the former is a more natural and important concept.

Lam's ideal $J_{\mathrm{L}, k}$ contains $\mathbb{Q}(q) \otimes_{\mathbb{Z}} I_{\mathrm{S}}$; this will follow from Proposition 4.11 below.

A word $\mathrm{v} \in \mathcal{U}^{*}$ is called a nonzero $k$-word if for every pair $i<j$ such that $\mathrm{v}_{i}=\mathrm{v}_{j}$, there exist $s, t$ satisfying $i<s<t<j$ and $\left\{\mathrm{v}_{s}, \mathrm{v}_{t}\right\}=\left\{\mathrm{v}_{i}-k, \mathrm{v}_{i}+k\right\}$. The relevance of this notion in our current context becomes clear from the following lemmas, which can be found in [5, Proposition-Definition 2.2 and Proposition 2.6(i)].

Lemma 4.7. A word $\mathbf{v}$ is a nonzero $k$-word if and only if $\mathbf{u}_{\mathrm{v}} \notin J_{\mathrm{L}, k}$.

Lemma 4.8. The nonzero $k$-words are precisely the words which appear in the sets $\mathrm{W}_{k}^{\prime}(\boldsymbol{\beta})$, as $\boldsymbol{\beta}$ ranges over all $k$-tuples of skew shapes.

Lam [22] used the above construction to obtain formulas (not manifestly positive) for the coefficients of Schur expansions of LLT polynomials. In our language, his result can be stated as follows.

Proposition 4.9. Let $\boldsymbol{\beta}$ be a $k$-tuple of skew shapes with shifted contents in $\{1, \ldots, N\}$. Then the corresponding new variant combinatorial LLT polynomial can be written as

$$
\mathcal{G}_{\boldsymbol{\beta}}(\mathbf{x} ; q)=F_{\gamma}(\mathbf{x})
$$

where

$$
\gamma=\sum_{\mathrm{v} \in \mathrm{W}_{k}^{\prime}(\boldsymbol{\beta})} q^{\mathrm{inv}_{k}^{\prime}(\mathrm{v})} \mathrm{v} \in J_{\mathrm{L}, k}^{\perp} .
$$

Consequently, $\mathfrak{c}_{\boldsymbol{\beta}}^{\lambda}(q)=\left\langle\mathfrak{J}_{\lambda}(\mathbf{u}), \gamma\right\rangle$. (Here we use the obvious modifications of our basic notions for $\gamma \in \mathbb{Q}(q) \otimes \mathcal{U}^{*}$.) 
Proof. The proof of the fact that $\gamma \in J_{\mathrm{L}, k}^{\perp}$ is exactly the same as the proof of the first statement of [6, Proposition 5.4] (even though the $\mathrm{W}_{k}^{\prime}(\boldsymbol{\beta})$ defined here is more general than that defined in [6]). The formula (4.10) is immediate from comparing (4.3) with (2.11). The last statement then follows from a straightforward modification of Proposition 2.9 to the coefficient field $\mathbb{Q}(q)$ (as opposed to working over $\mathbb{Z}$ ).

The switchboards studied in this paper were partially motivated by the D graphs of Assaf [1]. These graphs were introduced to give a combinatorial explanation of LLT and Macdonald positivity. We next explain some of Assaf's ideas using the language developed in Sections 2 and 3, and connect them to Lam's construction reviewed above.

Definition 4.10. The Assaf ideal $I_{\mathrm{A}, k}$ is the ideal in $\mathcal{U}$ generated by all monomials $\mathbf{u}_{\mathrm{w}} \in J_{\mathrm{L}, k}$ (cf. Lemma 4.7) together with the elements listed in (2.25)-(2.28).

It is easy to see that $I_{\mathrm{A}, k} \supset I_{\mathrm{R}, k} \supset I_{\mathrm{S}}$.

Proposition 4.11. $J_{\mathrm{L}, k} \supset \mathbb{Q}(q) \otimes_{\mathbb{Z}} I_{\mathrm{A}, k}$.

Proof. To ease notation in our argument, we identify each element $g \in I_{\mathrm{A}, k}$ with $1 \otimes g \in$ $\mathbb{Q}(q) \otimes_{\mathbb{Z}} I_{\mathrm{A}, k}$. It suffices to show that the generators of $I_{\mathrm{A}, k}$ vanish modulo $J_{\mathrm{L}, k}$. This clearly holds for (2.25)-(2.26), cf. (4.8). To check that the generators (2.27) vanish modulo $J_{\mathrm{L}, k}$, we compute, using (4.9) twice:

$$
u_{b} u_{a} u_{c} \equiv q u_{a} u_{b} u_{c} \equiv u_{a} u_{c} u_{b} \bmod J_{\mathrm{L}, k} \quad \text { for } c-a \leq k \text { and } a<b<c .
$$

The argument for the generators (2.28) is similar.

Proposition 4.11 implies that $I_{\mathrm{L}, k}=J_{\mathrm{L}, k} \cap \mathcal{U} \supset I_{\mathrm{A}, k}$.

Definition 4.12. An Assaf switchboard of level $k$ is a switchboard in which every vertex is a nonzero $k$-word, and every $i$-edge with endpoints $w$ and $w^{\prime}$ corresponds to a switch where $\left\{\mathrm{w}_{i-1} \mathrm{w}_{i} \mathrm{w}_{i+1}, \mathrm{w}_{i-1}^{\prime} \mathrm{w}_{i}^{\prime} \mathrm{w}_{i+1}^{\prime}\right\}$ fits one of the patterns

$$
\begin{aligned}
& \{\text { bac, bca }\} \text { or }\{\text { acb, cab }\}, \text { with } c-a>k \text { and } a<b<c ; \\
& \{\text { bac, acb }\} \text { or }\{\text { bca, cab }\} \text {, with } c-a \leq k \text { and } a<b<c .
\end{aligned}
$$

Assaf ideals $I_{\mathrm{A}, k}$ and Assaf switchboards are closely related, as the following proposition shows; its proof is straightforward.

Proposition 4.13. For a set of words $W$ of the same length, the following are equivalent:

- $\sum_{\mathrm{w} \in W} \mathrm{w} \in I_{\mathrm{A}, k}^{\perp}$;

- $W$ is the vertex set of an Assaf switchboard of level $k$.

If these conditions hold, then there is a unique Assaf switchboard with vertex set $W$.

The next result and its corollary relate Assaf switchboards to LLT polynomials.

Proposition 4.14. Let $t$ be a nonnegative integer, and $\boldsymbol{\beta}$ a $k$-tuple of skew shapes in which the shifted contents of all cells lie in $\{1, \ldots, N\}$. Then there is a unique level $k$ Assaf switchboard $\Gamma=\Gamma_{k}(\boldsymbol{\beta}, t)$ whose vertex set is $\left\{\mathrm{v} \in \mathrm{W}_{k}^{\prime}(\boldsymbol{\beta}) \mid \operatorname{inv}_{k}^{\prime}(\mathrm{v})=t\right\}$. 
Proof. Suppose that words $w$ and $w^{\prime}$ are related by a switch of the form (4.11) or (4.12). We must show that

$$
\begin{aligned}
& \mathrm{w} \in \mathrm{W}_{k}^{\prime}(\boldsymbol{\beta}) \text { if and only if } \mathrm{w}^{\prime} \in \mathrm{W}_{k}^{\prime}(\boldsymbol{\beta}) \\
& \operatorname{inv}_{k}^{\prime}(\mathrm{w})=\operatorname{inv}_{k}^{\prime}\left(\mathrm{w}^{\prime}\right) .
\end{aligned}
$$

Statement (4.13) is checked directly from the definition (4.2). Statement (4.14) is clear if $\mathrm{w}$ and $\mathrm{w}^{\prime}$ are related by a switch of the form (4.11). Suppose that $\left\{\mathrm{w}, \mathrm{w}^{\prime}\right\}=\{\mathrm{ybacz}, \mathrm{yacbz}\}$ for $c-a \leq k$ and $a<b<c$ and (sub)words y, z (the second case of (4.12) is similar).

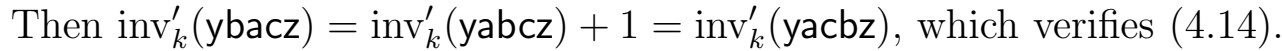

The uniqueness follows from Proposition 4.13,

Definition 4.15. LLT switchboards are the special Assaf switchboards $\Gamma_{k}(\boldsymbol{\beta}, t)$ described in Proposition 4.14. This terminology is justified by Corollary 4.16 below.

Corollary 4.16. Let $\boldsymbol{\beta}$ a k-tuple of skew shapes in which the shifted contents of cells lie in $\{1, \ldots, N\}$. Then the associated new variant combinatorial LLT polynomial is the $q$-generating function for the LLT switchboards $\Gamma_{k}(\boldsymbol{\beta}, t)$ :

$$
\mathcal{G}_{\boldsymbol{\beta}}(\mathbf{x} ; q)=\sum_{t} q^{t} F_{\Gamma_{k}(\boldsymbol{\beta}, t)}(\mathbf{x}) .
$$

Proof. This is a direct consequence of Propositions 4.9 and 4.14.

Remark 4.17. Corollary 4.16 is a restatement of the connection between certain $\mathrm{D}$ graphs and LLT polynomials described in [1, §4.2]. The switchboard $\bigsqcup_{t} \Gamma_{k}(\boldsymbol{\beta}, t)$ is, after relabeling vertices, the graph $\mathcal{G}_{c, D}^{(k)}$ defined in [1, $\left.\S 4.2\right]$, where $c$ is the content vector and $D$ is the $k$-descent set corresponding to $\boldsymbol{\beta}$ as in [1, Equation 4.2] (see also [5, Proposition 2.6]).

Example 4.18. Let $k=3, \boldsymbol{\beta}=(\square, \square, \square)=(2 / 1,1,2)$. Figure 6 shows all nonempty LLT switchboards $\Gamma_{3}(\boldsymbol{\beta}, t)$ and their symmetric functions $F_{\Gamma_{3}(\boldsymbol{\beta}, t)}$. Formula (4.15) then yields

$$
\begin{aligned}
& \mathcal{G}_{\boldsymbol{\beta}}(\mathbf{x} ; q)=s_{4}+q s_{31}+q^{2}\left(s_{31}+s_{22}\right)+q^{3} s_{211} . \\
& 1235 \\
& F_{\Gamma_{3}(\boldsymbol{\beta}, 0)}=s_{4} \\
& 2135 \stackrel{\tilde{2}}{-} 1325 \stackrel{\tilde{3}}{-} 1253 \\
& F_{\Gamma_{3}(\boldsymbol{\beta}, 1)}=s_{31} \\
& 3125 \stackrel{\tilde{2}}{-} 2315 \stackrel{3}{-} 2351 \\
& 2513 \stackrel{2}{=\frac{2}{3}} 2153 \\
& F_{\Gamma_{3}(\boldsymbol{\beta}, 2)}=s_{31}+s_{22} \\
& 2531 \stackrel{\tilde{2}}{\longrightarrow} 3251 \stackrel{3}{-} 3215 \\
& F_{\Gamma_{3}(\boldsymbol{\beta}, 3)}=s_{211}
\end{aligned}
$$

Figure 6: LLT switchboards $\Gamma_{3}(\boldsymbol{\beta}, t)$ for $\boldsymbol{\beta}=(2 / 1,1,2)$, and the corresponding symmetric functions $F_{\Gamma_{3}(\boldsymbol{\beta}, t)}$. The two connected components of $\Gamma_{3}(\boldsymbol{\beta}, 2)$ have symmetric functions $s_{31}$ (on the left) and $s_{22}$ (on the right). 
Example 4.19. Let $k=3, \boldsymbol{\beta}=(\square, \boxplus, \square)=(3 / 2,33 / 11,2 / 1)$. There are four nonempty LLT switchboards $\Gamma_{3}(\boldsymbol{\beta}, t)$, for $t=2,3,4,5$. (One of them is shown in Figure 7 .) The corresponding symmetric functions are:

$$
F_{\Gamma_{3}(\boldsymbol{\beta}, 2)}=s_{42}, \quad F_{\Gamma_{3}(\boldsymbol{\beta}, 3)}=s_{33}+s_{321}, \quad F_{\Gamma_{3}(\boldsymbol{\beta}, 4)}=s_{321}+s_{222}, \quad F_{\Gamma_{3}(\boldsymbol{\beta}, 5)}=s_{2211} .
$$

Hence by Corollary 4.16, the LLT polynomial $\mathcal{G}_{\boldsymbol{\beta}}(\mathbf{x} ; q)$ is given by

$$
\mathcal{G}_{\boldsymbol{\beta}}(\mathbf{x} ; q)=q^{2} s_{42}+q^{3}\left(s_{33}+s_{321}\right)+q^{4}\left(s_{321}+s_{222}\right)+q^{5} s_{2211} .
$$

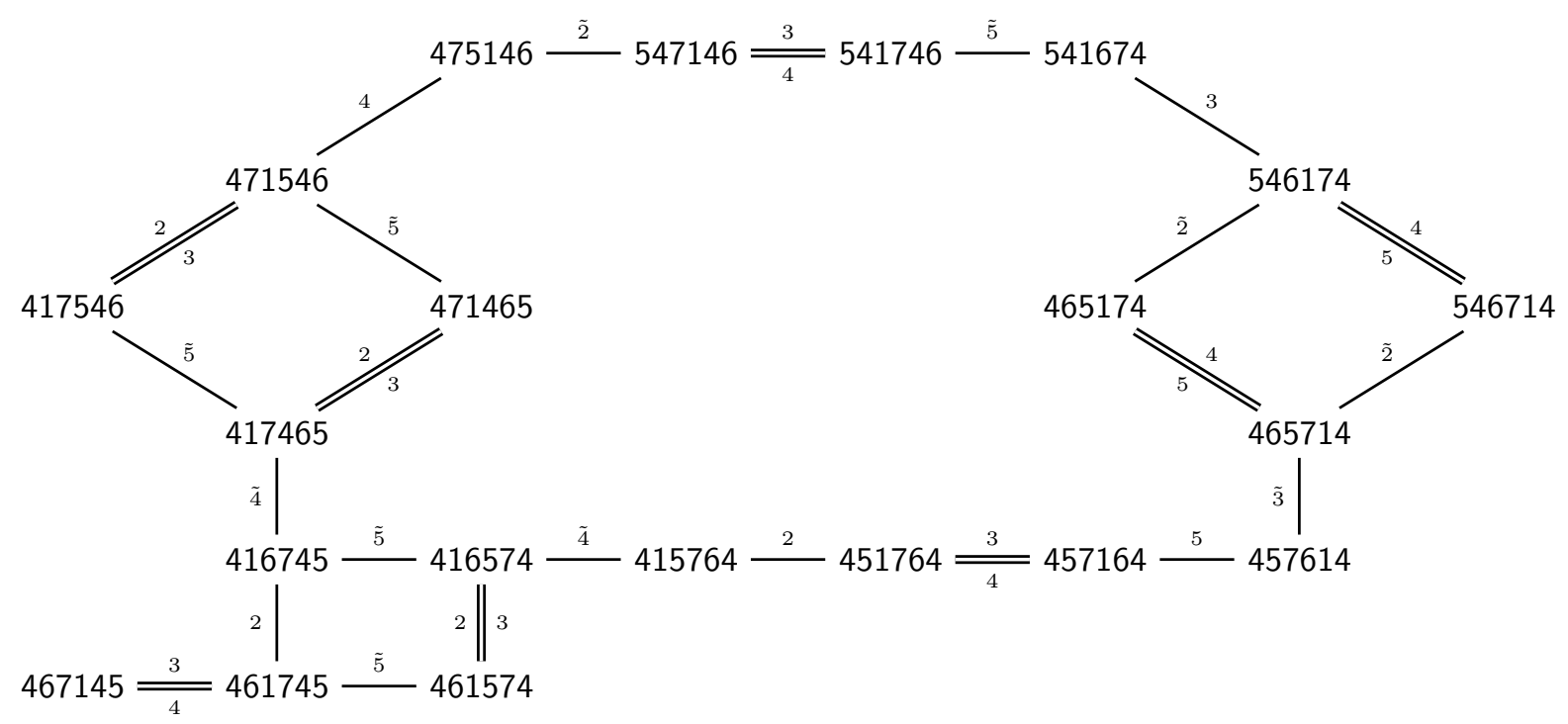

Figure 7: The LLT switchboard $\Gamma_{k}(\boldsymbol{\beta}, t)$, for $k=3, t=3$, and $\boldsymbol{\beta}=(3 / 2,33 / 11,2 / 1)$. The associated symmetric function is $F_{\Gamma_{3}(\boldsymbol{\beta}, 3)}=s_{33}+s_{321}$.

Definition 4.20. An Assaf symmetric function of level $k$ is the symmetric function $F_{\Gamma}(\mathbf{x})$ associated to an Assaf switchboard $\Gamma$ of level $k$.

Conjecture 4.21. Assaf symmetric functions are Schur positive.

Conjecture 4.21 is implicit in the work of Assaf, as is its slightly stronger variant Conjecture 2.16. (We opted for the latter variant in Section 2 primarily because it was much easier to formulate.) By Corollary 4.16, the coefficient of $q^{t}$ in an LLT polynomial is an Assaf symmetric function associated to a special Assaf switchboard $\Gamma_{k}(\boldsymbol{\beta}, t)$, so Conjecture 4.21 is a stronger statement than Schur positivity of LLT polynomials. In a sense, it is strictly stronger, as Example 4.18 illustrates: the $\operatorname{LLT}$ switchboard $\Gamma_{3}(\boldsymbol{\beta}, 2)$ in Figure 6 is disconnected, so $F_{\Gamma_{3}(\boldsymbol{\beta}, 2)}$ is a sum of two nonzero Assaf symmetric functions.

We conclude this section by recalling from [5] the main positive result of this approach to Schur positivity of LLT and Macdonald polynomials: a combinatorial description of the coefficients $\mathfrak{c}_{\boldsymbol{\beta}}^{\lambda}(q)$ (see (4.4)) in the special case $k=3$. Before doing so, we review earlier related work.

The LLT polynomial for $k=1$ is nothing but the skew Schur function for the corresponding shape. For $k=2$, an explicit combinatorial rule for the coefficients $\mathfrak{c}_{\boldsymbol{\beta}}^{\lambda}(q)$ was 
stated by Carré and Leclerc [8]; its proof was completed by van Leeuwen [34], cf. [16, §9]. Relatedly, Fishel [10] gave the first combinatorial interpretation for the coefficient of $s_{\lambda}(\mathbf{x})$ in the transformed Macdonald polynomial $\tilde{H}_{\mu}(\mathbf{x} ; q, t)$ in the case when $\mu$ has 2 columns (using rigged configurations). Alternative formulas for this case were given in [23, 36].

Conjecture 4.21 is known to hold in the following special cases (the results below are stronger than their counterparts in the previous paragraph, but came later): for $k=1$, the Assaf ideal $I_{\mathrm{A}, k}$ contains the plactic ideal (cf. Definition 5.4), hence for any connected component $\Gamma$ of an Assaf switchboard of level 1, the symmetric function $F_{\Gamma}$ is just a Schur function, cf. Proposition 5.6. Assaf [1, Theorem 4.9] showed that this is also true for connected Assaf switchboards of level 2. Hence Conjecture 4.21 holds for $k \in\{1,2\}$. Roberts [29, Theorem 4.11] extended the work of Assaf to a setting that contains the $k=2$ case, by proving that the symmetric function of any connected Assaf switchboard contained in $\Gamma_{k}(\boldsymbol{\beta}, t)$ is a Schur function whenever $\max _{i \in \mathbb{Z}}|C(\boldsymbol{\beta}) \cap[i, i+k]| \leq 3$ where $C(\boldsymbol{\beta})$ is the set of distinct shifted contents of the cells of $\boldsymbol{\beta}$ (cf. (4.1)). Assaf [1, Theorem 4.10] and Novelli-Schilling [28, Theorem 3] gave an explicit positive formula for the Schur expansion of the Assaf symmetric functions in the case $k \geq N-1$; note that in this case, Assaf switchboards have only rotation switches.

To state the main result of [5], we will need a couple of auxiliary notions. For a partition $\lambda$, let $\operatorname{RSST}(\lambda ; N)$ denote the set of semistandard Young tableaux $T$ of shape $\lambda$ and entries in $\{1, \ldots, N\}$ satisfying the following constraints:

- the entries strictly increase across the rows and down the columns;

- the entries increase in increments of at least 3 along diagonals.

For $T \in \operatorname{RSST}(\lambda ; N)$, we produce a word $\operatorname{sqread}(T) \in \mathcal{U}^{*}$ by reading the entries of $T$ in the following order. Let us circle each entry $c$ of $T$ such that the entry immediately west of it is $c-1$. The word sqread $(T)$ is then obtained by reading the diagonals of $T$ one by one, starting from the southwest corner and finishing at the northeast corner. In each diagonal, we first read the circled entries going northwest, then the uncircled entries going southeast. To illustrate, the tableau

$$
T=\begin{array}{|l|l|l|l|}
\hline 1 & 2 & 4 & 6 \\
\hline 3 & 4 & 5 & 7 \\
\hline 5 & 6 & 8 & 9
\end{array} \text { has circled entries } \begin{array}{|l|l|l|l|l|}
\hline 1 & 2 & 4 & 6 \\
\hline 3 & 4 & 5 & 7 \\
\hline 5 & 6 & 8 & 9 \\
\hline
\end{array} \quad \text { and } \operatorname{sqread}(T)=563418952476 .
$$

Theorem 4.22 ([5, Theorem 1.1]). For any partition $\lambda$, the noncommutative Schur function $\mathfrak{J}_{\lambda}(\mathbf{u})$ is $\mathbb{Z}$-monomial positive modulo $J_{\mathrm{L}, 3}$. A monomial expansion is given by

$$
\mathfrak{J}_{\lambda}(\mathbf{u}) \equiv \sum_{T \in \operatorname{RSST}(\lambda ; N)} \mathbf{u}_{\text {sqread }(T)} \bmod J_{\mathrm{L}, 3} .
$$

Theorem 4.22 combined with Proposition 4.9 yields the explicit combinatorial formula

$$
\mathfrak{c}_{\boldsymbol{\beta}}^{\lambda}(q)=\sum_{\substack{T \in \operatorname{RSST}(\lambda ; N) \\ \operatorname{sqread}(T) \in \mathrm{W}_{3}^{\prime}(\boldsymbol{\beta})}} q^{\operatorname{inv}_{3}^{\prime}(\operatorname{sqread}(T))}
$$

for the coefficients in the Schur expansion of an LLT polynomial indexed by a 3-tuple of skew shapes; see [5, Corollary 4.3] for further details. Cf. also Conjecture [7.9. 


\section{Plactic, Niplactic, AND Beyond}

In this section, we recast some of the key results obtained in [11] in the language of ideals and switchboards. To this end, we introduce some notation. First, we set

$$
I_{Q}=I_{\mathrm{B}}+I_{\mathrm{S}}
$$

Equivalently, $I_{\bigotimes}$ is the ideal in $\mathcal{U}$ generated by the elements (2.21) $-(2.22)$ and (2.29) $-(2.31)$.

Let $\operatorname{SSYT}(\lambda ; N)$ denote the set of semistandard Young tableaux of shape $\lambda$ and entries from $\{1, \ldots, N\}$. The column reading word $\operatorname{colword}(T) \in \mathcal{U}^{*}$ of a tableau $T \in \operatorname{SSYT}(\lambda ; N)$ is the word obtained by concatenating the columns of $T$ (reading each column bottom to top), starting with the leftmost column. To illustrate, the tableau

$$
T=\begin{array}{|l|l|l|l|}
\hline 1 & 1 & 2 & 2 \\
\hline 2 & 2 & 3 & \\
\hline 5 & 6 & 7 \\
\hline
\end{array}
$$

of shape $\lambda=433$ has column reading word colword $(T)=5216217322$.

Theorem 5.1 ([1] $)$. The noncommutative Schur functions $\mathfrak{J}_{\lambda}(\mathbf{u})$ are $\mathbb{Z}$-monomial positive modulo the ideal $I_{Q}$. Explicitly,

$$
\mathfrak{J}_{\lambda}(\mathbf{u}) \equiv \sum_{T \in \operatorname{SSYT}(\lambda ; N)} \mathbf{u}_{\operatorname{colword}(T)} \bmod I_{Q} .
$$

Recall that Theorem 2.14 of this paper strengthens Theorem 5.1 by replacing $I_{Q}$ by $I_{\mathrm{B}}$.

Definition 5.2. A switchboard $\Gamma$ is called rotation-free if it has no rotation switches. That is, every switch in $\Gamma$ is Knuth, braid, or idempotent, see Definition 3.1 and Figure 2

Rotation-free switchboards are directly related to the ideal $I_{\mathbb{Q}}$ : it is not hard to check that a $(0,1)$-vector in $I_{Q}^{\perp}$ is the same as the sum of vertices of a rotation-free switchboard. Combining this with Theorem 5.1 and Proposition 2.9, we obtain the following corollary.

Corollary 5.3. For a rotation-free switchboard $\Gamma$, the symmetric function $F_{\Gamma}(\mathbf{x})$ is Schur positive. The coefficient of $s_{\lambda}(\mathbf{x})$ in $F_{\Gamma}(\mathbf{x})$ is the number of tableaux $T \in \operatorname{SSYT}(\lambda ; N)$ such that the column reading word colword $(T)$ appears as a vertex in $\Gamma$.

Several important families of symmetric functions, some of which are reviewed below (see [11] for additional examples), arise from ideals containing $I_{\mathbb{Q}}$. For each of these families, Corollary 5.3 provides a manifestly positive combinatorial rule for the coefficients in the corresponding Schur expansions.

The following classical construction goes back to Lascoux and Schützenberger [25].

Definition 5.4. The plactic ideal $I_{\text {plac }} \subset \mathcal{U}$ is generated by the "Knuth elements"

$$
\begin{array}{ll}
u_{a} u_{c} u_{b}-u_{c} u_{a} u_{b} & \text { for } a \leq b<c ; \\
u_{b} u_{a} u_{c}-u_{b} u_{c} u_{a} & \text { for } a<b \leq c .
\end{array}
$$

It is easy to see that $I_{\text {plac }} \supset I_{Q}$. 
Definition 5.5. A switchboard is called plactic if all its switches are Knuth. A connected plactic switchboard has the words in a Knuth (= plactic) equivalence class as its vertices, and Knuth switches as its edges. For a semistandard Young tableau $T$, we denote by $\Gamma_{T}$ the switchboard whose vertex set consists of words with insertion tableau $T$.

The $(0,1)$-vectors in $I_{\text {plac }}^{\perp}$ are the same as sums of distinct Knuth equivalence classes.

The switchboards $\Gamma_{T}$ have been studied implicitly in the huge body of work on the Robinson-Schensted-Knuth correspondence and jeu de taquin. They were studied explicitly in the context of $W$-graphs [20, 33], and their combinatorial structure was thoroughly investigated in [1, 29]. It is well known that the edge-labeled graph $\Gamma_{T}$, after forgetting vertex labels, depends only on the shape of $T$ and not on its entries; these edge-labeled graphs (with slightly different conventions) are the standard dual equivalence graphs of [1].

The symmetric functions associated with connected plactic switchboards are nothing but Schur functions. This is just a restatement of the well known expansion of a Schur polynomial in terms of Gessel's fundamental quasisymmetric functions:

Proposition 5.6 ([14], [32, Chapter 7]). For $T \in \operatorname{SSYT}(\lambda ; N)$, we have $F_{\Gamma_{T}}(\mathbf{x})=s_{\lambda}(\mathbf{x})$.

We next discuss the Stanley symmetric functions, also known as stable Schubert polynomials. They are related to the following ideal [12].

Definition 5.7. The nilCoxeter ideal $I_{\mathrm{nCox}}$ of $\mathcal{U}$ is generated by the elements

$$
\begin{array}{ll}
u_{a}^{2} & \text { for all } a ; \\
u_{a} u_{c}-u_{c} u_{a} & \text { for } c-a \geq 2 ; \\
u_{a} u_{b} u_{a}-u_{b} u_{a} u_{b} & \text { for } b-a=1 .
\end{array}
$$

It is easy to see that $I_{\mathrm{nCox}} \supset I_{Q}$.

The monomials $\mathbf{u}_{\mathrm{w}} \notin I_{\mathrm{nCox}}$ are labeled by reduced words $\mathrm{w}$ for the symmetric group $\mathcal{S}_{N+1}$ (viewed as words in $\mathcal{U}^{*}$ ). Moreover $\mathbf{u}_{\mathrm{v}} \equiv \mathbf{u}_{\mathrm{w}} \bmod I_{\mathrm{nCox}}$ if and only if $\mathrm{v}$ and $\mathrm{w}$ are reduced words for the same permutation $\pi \in \mathcal{S}_{N+1}$.

We denote by $\operatorname{Red}(\pi)$ the set of reduced words for $\pi \in \mathcal{S}_{N+1}$, and set

$$
\gamma(\pi)=\sum_{w \in \operatorname{Red}(\pi)} w \in \mathcal{U}^{*}
$$

The $(0,1)$-vectors $\gamma(\pi)$ form a $\mathbb{Z}$-basis of $I_{\mathrm{nCox}}^{\perp}$.

The Stanley symmetric function associated to the permutation $\pi \in \mathcal{S}_{N+1}$ is defined by

$$
F_{\gamma(\pi)}(\mathbf{x})=\sum_{\mathrm{w} \in \operatorname{Red}(\pi)} Q_{\operatorname{Des}(\mathrm{w})}(\mathbf{x})
$$

By Corollary 5.3, $F_{\gamma(\pi)}(\mathbf{x})$ is a Schur positive symmetric function [9]:

Corollary 5.8. The coefficient of $s_{\lambda}(\mathbf{x})$ in $F_{\gamma(\pi)}(\mathbf{x})$ is the number of tableaux $T$ of shape $\lambda$ whose column reading word colword $(T)$ is a reduced word for $\pi$. 
Definition 5.9. The nilplactic ideal $I_{\text {nplac }}$ of $\mathcal{U}$ is generated by the elements

$$
\begin{array}{ll}
u_{a}^{2} & \text { for all } a ; \\
u_{a} u_{c} u_{a} & \text { for } c-a \geq 2 ; \\
u_{c} u_{a} u_{c} & \text { for } c-a \geq 2 ; \\
u_{b} u_{a} u_{c}-u_{b} u_{c} u_{a} & \text { for } a<b<c ; \\
u_{a} u_{c} u_{b}-u_{c} u_{a} u_{b} & \text { for } a<b<c ; \\
u_{a} u_{b} u_{a}-u_{b} u_{a} u_{b} & \text { for } b-a=1 .
\end{array}
$$

It is easy to check that $I_{\text {nCox }} \supset I_{\text {nplac }} \supset I_{Q}$. A switchboard $\Gamma$ is called nilplactic if

- all switches in $\Gamma$ are either Knuth switches on 3 distinct letters, or braid switches;

- no word in $\Gamma$ contains the same letter twice in succession;

- no word in $\Gamma$ contains 3 consecutive letters aca or cac with $c-a \geq 2$.

The vertices of a connected nilplactic switchboard form a nilplactic equivalence class; they are reduced words for the same permutation. The $(0,1)$-vectors in $I_{\text {nplac }}^{\perp}$ are sums of distinct niplactic classes.

It is known [9, 26] that the symmetric function $F_{\Gamma}$ associated to any connected nilplactic switchboard $\Gamma$ is a Schur function. Moreover any connected nilplactic switchboard is isomorphic, as an edge-labeled graph, to one of the standard dual equivalence graphs.

Nilplactic switchboards give another way of understanding the Schur expansions of Stanley symmetric functions. The set $\operatorname{Red}(\pi)$ of reduced words for a permutation $\pi \in$ $\mathcal{S}_{N+1}$ is the vertex set of a unique (generally disconnected) nilplactic switchboard $\Gamma(\pi)$. Hence the coefficient of $s_{\lambda}(\mathbf{x})$ in $F_{\gamma(\pi)}(\mathbf{x})=F_{\Gamma(\pi)}(\mathbf{x})$ is the number of components of $\Gamma(\pi)$ whose associated symmetric function is $s_{\lambda}(\mathbf{x})$. An example is given in Figure 8 ,

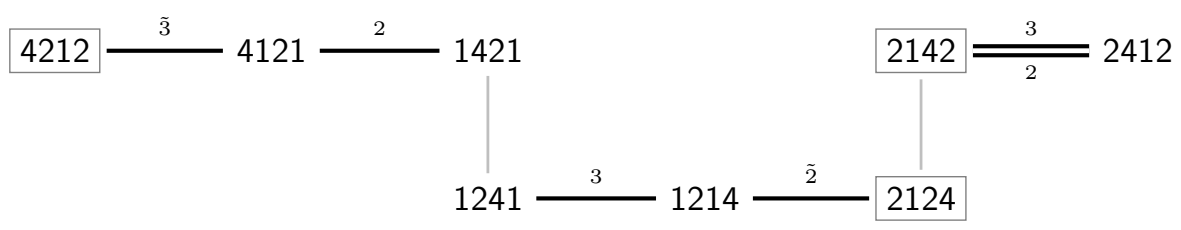

Figure 8: Let $\pi=32154 \in \mathcal{S}_{5}$. Without the vertical edges, this is the nilplactic switchboard $\Gamma(\pi)$ on the vertex set $\operatorname{Red}(\pi)$. Vertical edges correspond to relations ac $\sim$ ca which are not switches. The associated symmetric function is the Stanley symmetric function $F_{\Gamma(\pi)}=s_{31}+s_{22}+s_{211}$. By Corollary 5.8, the Schur expansion of $F_{\Gamma(\pi)}$ is determined by the reduced words which are column reading words of tableaux.

Definition 5.10. Let $I_{\mathrm{H}}$ be the ideal in $\mathcal{U}$ generated by the elements

$$
\begin{array}{ll}
u_{b} u_{a} u_{c}-u_{b} u_{c} u_{a} & \text { for } a<b<c ; \\
u_{a} u_{c} u_{b}-u_{c} u_{a} u_{b} & \text { for } a<b<c ; \\
u_{a} u_{b} u_{a}-u_{b} u_{a} u_{b} & \text { for } b-a=1 ; \\
u_{b} u_{b} u_{a}-u_{b} u_{a} u_{a} & \text { for } b-a=1 .
\end{array}
$$


It is easy to check that $I_{\text {nplac }} \supset I_{\mathrm{H}} \supset I_{Q}$. Applying Corollary 5.3 to certain switchboards associated with $I_{\mathrm{H}}$ yields the Schur expansions of stable Grothendieck polynomials, up to a predictable sign; see [11, Corollary 4.2] for details. We note that connected components of these switchboards do not have to be standard dual equivalence graphs; this sets them apart from connected plactic or nilplactic switchboards. An example is given in Figure 9 .

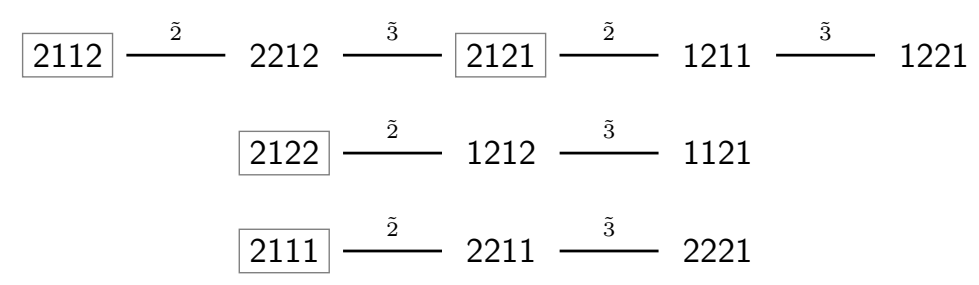

Figure 9: Each component (hence their union) is a switchboard whose vertex sum lies in $I_{\mathrm{H}}^{\perp}$. Their symmetric functions are (top to bottom): $s_{31}+s_{22}, s_{31}, s_{31}$. By Corollary 5.3, these Schur expansions can be read off from the vertices that are column reading words.

\section{Semimatched ideals}

Definition 6.1. Let us partition the set of 3 -element subsets of $\{1, \ldots, N\}$ into three categories, by declaring each triple $S \subset\{1, \ldots, N\}$ to be either a Knuth triple, or a rotation triple, or an undecided triple. Similarly, declare each subset $\{a, a+1\} \subset\{1, \ldots, N\}$ to be a Knuth pair, a braid/idempotent pair, or an undecided pair. Let $I_{M}$ be a monomial ideal in $\mathcal{U}$. The semimatched ideal $I$ associated to this data is the ideal generated by $I_{M}$, the switchboard ideal $I_{\mathrm{S}}$ (whose generators are (2.29)-(2.32) ), and the elements listed below:

$$
\begin{array}{ll}
u_{a} u_{c} u_{b}-u_{c} u_{a} u_{b} & \text { if } a<b<c \text { is a Knuth triple, } \\
u_{b} u_{a} u_{c}-u_{b} u_{c} u_{a} & \text { if } a<b<c \text { is a Knuth triple, } \\
u_{a} u_{c} u_{b}-u_{b} u_{a} u_{c} & \text { if } a<b<c \text { is a rotation triple, } \\
u_{c} u_{a} u_{b}-u_{b} u_{c} u_{a} & \text { if } a<b<c \text { is a rotation triple, } \\
u_{a} u_{b} u_{a}-u_{b} u_{a} u_{a} & \text { if } b-a=1 \text { and }\{a, b\} \text { is a Knuth pair, } \\
u_{b} u_{a} u_{b}-u_{b} u_{b} u_{a} & \text { if } b-a=1 \text { and }\{a, b\} \text { is a Knuth pair, } \\
u_{a} u_{b} u_{a}-u_{b} u_{a} u_{b} & \text { if } b-a=1 \text { and }\{a, b\} \text { is a braid/idempotent pair, } \\
u_{b} u_{a} u_{a}-u_{b} u_{b} u_{a} & \text { if } b-a=1 \text { and }\{a, b\} \text { is a braid/idempotent pair. }
\end{array}
$$

For a semimatched ideal $I$ as above, an $I$-switchboard is a switchboard in which every vertex $\mathrm{w}$ corresponds to a monomial $u_{\mathrm{w}} \notin I_{M}$, and every edge corresponds to a switch that fits one of the patterns listed below:

$\{$ bac, bca $\}$ or $\{\mathrm{acb}, \mathrm{cab}\}$ for Knuth triples and undecided triples $a<b<c$;

$\{\mathrm{bac}, \mathrm{acb}\}$ or $\{\mathrm{bca}, \mathrm{cab}\}$ for rotation triples and undecided triples $a<b<c$;

$\{$ aba, baa $\}$ or $\{$ bab, bba $\}$ for $b-a>1$;

$\{$ aba, baa $\}$ or $\{$ bab, bba $\}$ for Knuth and undecided pairs $\{a, b\}(b-a=1)$;

$\{$ aba, bab $\}$ or $\{$ baa, bba $\}$ for braid/idempotent and undecided pairs $(b-a=1)$. 
In Figure 1, the following ideals are semimatched: $I_{\mathrm{S}}, I_{Q}, I_{\mathrm{R}, k}, I_{\mathrm{A}, k}, I_{\mathrm{plac}}, I_{\mathrm{H}}$, and $I_{\mathrm{nplac}}$. For example, the ideal $I_{Q}=I_{\mathrm{B}}+I_{\mathrm{S}}$ (cf. (5.1) ) is the semimatched ideal in which all triples are Knuth, all pairs undecided, and $I_{M}=\{0\}$. The Assaf ideal $I_{\mathrm{A}, k}$ is the semimatched ideal with Knuth triples $a<b<c$ for $c-a>k$, rotation triples $a<b<c$ for $c-a \leq k$, no undecided triples, all pairs Knuth, and $I_{M}$ generated by the monomials in $J_{\mathrm{L}, k}$.

We observe that Assaf switchboards are the same as $I_{\mathrm{A}, k}$-switchboards, rotation-free switchboards are $I_{Q^{-}}$-switchboards, plactic switchboards are $I_{\text {plac-switchboards, and nilplac- }}$

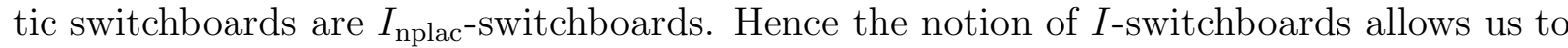
recover the classes of switchboards studied in Sections 45 from their corresponding ideals.

Proposition 6.2. Let I be a semimatched ideal. For a set of words $W$ of the same length, the following are equivalent:

- $\sum_{\mathrm{w} \in W} \mathrm{w} \in I^{\perp}$;

- $W$ is the vertex set of an I-switchboard.

Definition 6.3. A triples ideal is a semimatched ideal in which there are no undecided triples, all pairs $\{a, a+1\}$ are Knuth pairs, and $I_{M}=\{0\}$. Hence the data defining a triples ideal is a map from the set of triples in $\{1, \ldots, N\}$ to the 2-element set $\{$ Knuth, rotation . A triples switchboard is an $I$-switchboard associated to a triples ideal $I$. A triples $\mathrm{D}_{0}$ graph is a triples switchboard whose words have no repeated letters; these were studied in [6], see [6, Example 2.5 and Definition 7.13].

Example 6.4. Let $\Gamma$ be the triples switchboard on the vertex set $\mathcal{S}_{5}$ corresponding to the triples ideal in which 124, 245, and 345 are rotation triples and the rest are Knuth triples. The components of $\Gamma$ have symmetric functions $s_{41}+s_{32}+s_{311}+s_{221}$ (see Figure 10), $s_{32}+s_{311}+s_{221}+s_{2111}, s_{41}+s_{32}, s_{221}+s_{2111}, s_{5}, s_{41}$ (two components), $s_{32}$ (two), $s_{311}$ (four), $s_{221}$ (two), $s_{2111}$ (two), and $s_{11111}$.

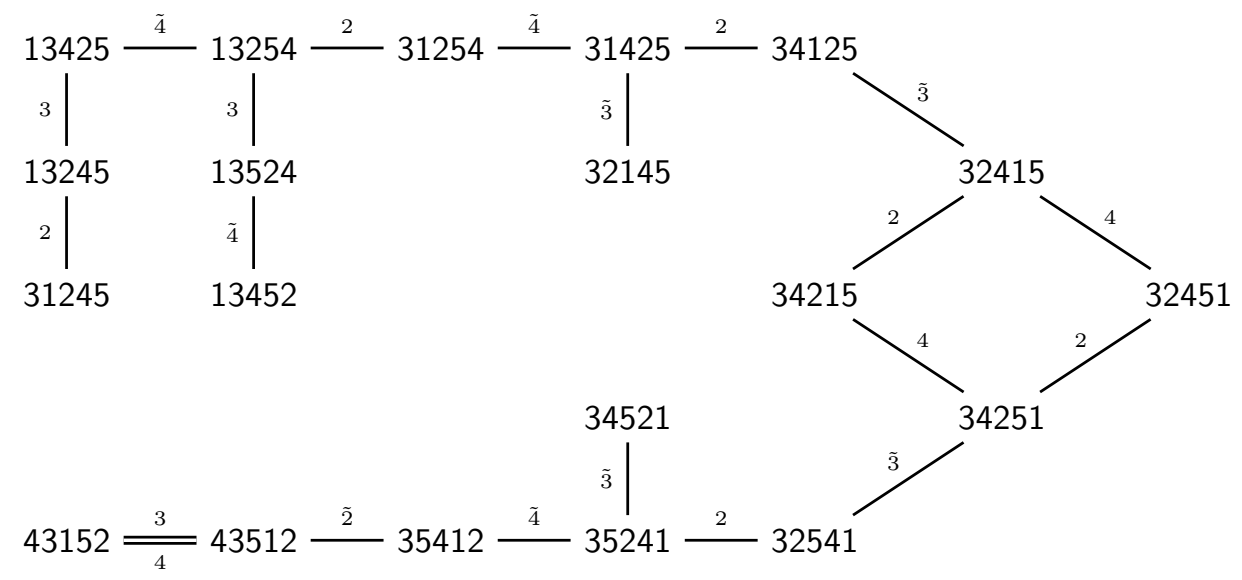

Figure 10: A triples switchboard for the triples ideal in which 124, 245, and 345 are rotation triples and all other triples are Knuth. See Example 6.4.

Problem 6.5. Is the symmetric function $F_{\Gamma}$ Schur positive for any triples switchboard $\Gamma$ ?

If $\Gamma$ is a $\mathrm{D}_{0}$ graph with $N \leq 5$, then $F_{\Gamma}$ is Schur positive by [6, Corollary 4.9]. Extensive computer tests provide supporting evidence for Schur positivity for $N \in\{6,7,8\}$. 


\section{The SChur POSITIVITy THREShold}

As of this writing, the following tantalizing questions remain unanswered: For which ideals $I$ are the symmetric functions $F_{\gamma}(\mathbf{x})$ Schur positive for all vectors $\gamma \in \mathcal{U}_{\geq 0}^{*} \cap I^{\perp}$ ? For which $I$ are the noncommutative Schur functions $\mathfrak{J}_{\lambda}(\mathbf{u})$ monomial positive modulo $I$ ? In this section, we assemble our best attempts towards answers, including known special cases (cf. Figure 11) and strategies for further exploration. We believe it particularly instructive to examine the "phase transition" between Schur positivity and non-positivityfor instance, by looking at two ideals which are "as close as possible" while requiring $\mathfrak{J}_{\lambda}(\mathbf{u})$ be monomial positive modulo one but not the other.

Here is one specific line of inquiry. Given ideals $I_{1}, \ldots, I_{m}$ for which monomial positivity of $\mathfrak{J}_{\lambda}(\mathbf{u})$ is known (or expected) to hold modulo each $I_{j}$, is there a monomial positive expression for $\mathfrak{J}_{\lambda}(\mathbf{u})$ which is true modulo each ideal $I_{j}$, and consequently modulo the intersection $\bigcap I_{j}$ ?

To simplify calculations, we prefer to work with ideals which contain both the switchboard ideal $I_{\mathrm{S}}$ and the ideal $I_{\text {st }}$ generated by monomials with repeated entries. This translates into restricting from switchboards to $\mathrm{D}_{0}$ graphs, see Definitions 3.13 3.14.

We begin by looking more closely at the counterexample in Figure 5. For $N=6$, the noncommutative Schur function $\mathfrak{J}_{(2,2,2)}(\mathbf{u})$ is given by (cf. (2.12))

$$
\mathfrak{J}_{(2,2,2)}(\mathbf{u})=e_{3}(\mathbf{u})^{2}-e_{4}(\mathbf{u}) e_{2}(\mathbf{u}) \equiv \sum_{\substack{w \in \mathcal{S}_{6} \\ \operatorname{Des}(\mathrm{w})=\{1,2,4,5\}}} \mathbf{u}_{\mathrm{w}}-\sum_{\substack{\mathrm{w} \in \mathcal{S}_{6} \\ \operatorname{Des}(\mathrm{w})=\{1,2,3,5\}}} \mathbf{u}_{\mathrm{w}} \bmod I_{\mathrm{st}}
$$

Note that in the last expression, the two sums have 20 and 15 monomials, respectively. Let us see how this formula simplifies modulo some ideals.

Proposition 7.1. Let $N=6$. Modulo the ideal $I_{\mathrm{S}}+I_{\mathrm{st}}$, the noncommutative Schur function $\mathfrak{J}_{(2,2,2)}(\mathbf{u})$ cannot be written as a sum of 5 monomials, nor as a sum of 6 monomials minus a monomial. It can be written as a sum of 7 monomials minus 2 monomials:

$$
\begin{aligned}
\mathfrak{J}_{(2,2,2)}(\mathbf{u}) \equiv \mathbf{u}_{321654}+\mathbf{u}_{426513}+\mathbf{u}_{562143}+\mathbf{u}_{436512}+\mathbf{u}_{563412} & +\mathbf{u}_{462315}+\mathbf{u}_{452316} \\
& -\mathbf{u}_{462351}-\mathbf{u}_{452361}
\end{aligned}
$$

Modulo the ideal $\bigcap_{k}\left(I_{\mathrm{A}, k}+I_{\mathrm{st}}\right)$, the noncommutative Schur function $\mathfrak{J}_{(2,2,2)}(\mathbf{u})$ has exactly four $\mathbb{Z}$-monomial positive expressions:

$$
\begin{aligned}
\mathfrak{J}_{(2,2,2)}(\mathbf{u}) & \equiv \mathbf{u}_{321654}+\mathbf{u}_{426513}+\mathbf{u}_{562143}+\mathbf{u}_{431652}+\mathbf{u}_{563412} \\
& \equiv \mathbf{u}_{321654}+\mathbf{u}_{462153}+\mathbf{u}_{521643}+\mathbf{u}_{436512}+\mathbf{u}_{563412} \\
& \equiv \mathbf{u}_{321654}+\mathbf{u}_{462513}+\mathbf{u}_{521643}+\mathbf{u}_{436152}+\mathbf{u}_{563412} \\
& \equiv \mathbf{u}_{321654}+\mathbf{u}_{462513}+\mathbf{u}_{526143}+\mathbf{u}_{431652}+\mathbf{u}_{563412}
\end{aligned}
$$

Remark 7.2. We feel that in this example, the ideals $I_{\mathrm{S}}+I_{\mathrm{st}}$ and $\bigcap_{k}\left(I_{\mathrm{A}, k}+I_{\mathrm{st}}\right)$ are very close to (but on different sides of) the threshold where Schur positivity breaks. Formula (7.1), with only two minus signs, suggests that $\mathfrak{J}_{(2,2,2)}(\mathbf{u})$ is quite close to being monomial positive modulo $\left(I_{\mathrm{S}}+I_{\mathrm{st}}\right)$. At the same time, the fact that there are only four monomial positive expressions for $\mathfrak{J}_{(2,2,2)}(\mathbf{u})$ modulo $\bigcap_{k}\left(I_{\mathrm{A}, k}+I_{\mathrm{st}}\right)$ suggests that monomial positivity 
just barely holds here. Indeed, for an ideal $I$ generated by monomials and binomials of the form $\mathbf{u}_{\mathfrak{v}}-\mathbf{u}_{\mathrm{w}}$, the number of monomial positive expressions for $\mathfrak{J}_{\lambda}(\mathbf{u})$ modulo $I$ (assuming they exist) is the product of the sizes of the equivalence classes mod $I$ to which these monomials belong (assuming those equivalence classes are distinct). This number is typically quite large, e.g., it is equal to $5^{5}$ for the plactic ideal in this example, cf. the proof below.

Proof of Proposition 7.1. We first describe a (somewhat brute force) alternative way of showing that $\mathfrak{J}_{(2,2,2)}(\mathbf{u})$ is not $\mathbb{Z}$-monomial positive modulo $I_{\mathrm{S}}+I_{\text {st }}$ (which implies the same for $I_{\mathrm{S}}$, cf. Corollary [3.9(ii)). Assume the contrary, i.e.,

$$
\mathfrak{J}_{(2,2,2)}(\mathbf{u}) \equiv \mathbf{u}_{\mathrm{w}^{1}}+\mathbf{u}_{\mathrm{w}^{2}}+\mathbf{u}_{\mathrm{w}^{3}}+\mathbf{u}_{\mathrm{w}^{4}}+\mathbf{u}_{\mathrm{w}^{5}} \bmod \left(I_{\mathrm{S}}+I_{\mathrm{st}}\right)
$$

for some monomials $\mathbf{u}_{\mathrm{w}^{1}}, \ldots, \mathbf{u}_{\mathrm{w}^{5}} \in \mathcal{U}$. Then the same congruence also holds modulo $I_{\text {plac }}+I_{\text {st }} \supset I_{\mathrm{S}}+I_{\text {st }}$, where $I_{\text {plac }}$ is the plactic ideal from Definition 5.4. Since $I_{\text {plac }}+I_{\text {st }} \supset I_{Q}$, Theorem 5.1 says that

$$
\mathfrak{J}_{(2,2,2)}(\mathbf{u}) \equiv \sum_{T \in \operatorname{SSYT}(\lambda ; 6)} \mathbf{u}_{\operatorname{colword}(T)} \equiv \sum_{T \in \operatorname{SYT}(\lambda ; 6)} \mathbf{u}_{\operatorname{colword}(T)} \bmod \left(I_{\text {plac }}+I_{\text {st }}\right),
$$

where $\operatorname{SYT}(\lambda ; N)$ is the set of standard Young tableaux of shape $\lambda=(2,2,2)$. In order for the right-hand sides of (7.3) and (7.4) to be congruent to each other $\bmod \left(I_{\mathrm{plac}}+I_{\mathrm{st}}\right)$, the five words $w^{1}, \ldots, w^{5}$ must belong (in some order) to the Knuth equivalence classes of the five standard Young tableaux of shape $(2,2,2)$. Each of these equivalence classes consists of 5 words (indeed, permutations of $1,2,3,4,5,6)$. We then checked, using a noncommutative Gröbner basis calculation in Magma [7], that none of these $5^{5}$ possibilities is congruent to $\mathfrak{J}_{(2,2,2)}(\mathbf{u})$ modulo $I_{\mathrm{S}}+I_{\mathrm{st}}$.

A similar brute force calculation shows that there is no expression for $\mathfrak{J}_{(2,2,2)}(\mathbf{u})$ as a sum of six monomials minus another monomial that holds modulo $I_{\mathrm{S}}+I_{\mathrm{st}}$. Further calculations produce expressions with only two minus signs, including (7.1).

The second part of the proposition is established by a noncommutative Gröbner basis calculation similar to that above: for each of the $5^{5}$ expressions as in (7.3), we checked whether it holds modulo $\bigcap_{k}\left(I_{\mathrm{A}, k}+I_{\mathrm{st}}\right)$. Just as the argument above used $I_{\text {plac }}+I_{\mathrm{st}} \supset$ $I_{\mathrm{S}}+I_{\mathrm{st}}$, this one relies on the inclusion $I_{\mathrm{plac}}+I_{\mathrm{st}}=I_{\mathrm{A}, 1}+I_{\mathrm{st}} \supset \bigcap_{k}\left(I_{\mathrm{A}, k}+I_{\mathrm{st}}\right)$.

Remark 7.3. To better understand the transition from monomial positivity to nonpositivity, it is instructive to see how the expression in (7.1) collapses to (7.2) once we impose the relations corresponding to $\bigcap_{k}\left(I_{\mathrm{A}, k}+I_{\mathrm{st}}\right)$. Let us rewrite (7.1) as

$\mathfrak{J}_{(2,2,2)}(\mathbf{u}) \equiv \mathbf{u}_{321654}+\mathbf{u}_{426513}+\mathbf{u}_{562143}+\mathbf{u}_{436512}+\mathbf{u}_{563412}+\mathbf{u}_{4623[1,5]}+\mathbf{u}_{4523[1,6]} \bmod \left(I_{\mathrm{S}}+I_{\mathrm{st}}\right)$

where we used the notation

$$
\mathbf{u}_{\mathrm{v}[\mathrm{a}, \mathrm{b}] \mathrm{w}}=\mathbf{u}_{\mathrm{v}} u_{a} u_{b} \mathbf{u}_{\mathrm{w}}-\mathbf{u}_{\mathrm{v}} u_{b} u_{a} \mathbf{u}_{\mathrm{w}} .
$$

Consider the term $\mathbf{u}_{4523[1,6]}$. If $k \geq 5$, then $\mathbf{u}_{4523[1,6]} \equiv \mathbf{u}_{4352[1,6]} \bmod \left(I_{\mathrm{A}, k}+I_{\mathrm{st}}\right)$. If $k<5$, then $\mathbf{u}_{4523[1,6]} \equiv 0 \equiv \mathbf{u}_{4352[1,6]} \bmod \left(I_{\mathrm{A}, k}+I_{\mathrm{st}}\right)$. Hence, computing here and below modulo $\bigcap_{k}\left(I_{\mathrm{A}, k}+I_{\mathrm{st}}\right)$, we get $\mathbf{u}_{4523[1,6]} \equiv \mathbf{u}_{4352[1,6]} \equiv \mathbf{u}_{43[1,6] 52}$. Similar arguments yield $\mathbf{u}_{4623[1,5]} \equiv$ 
$\mathbf{u}_{4362[1,5]} \equiv \mathbf{u}_{436[1,5] 2}$. Consequently the difference of (17.1) and (7.2) is equal to

$$
\begin{aligned}
\mathbf{u}_{436512}+\mathbf{u}_{4623[1,5]} & +\mathbf{u}_{4523[1,6]}-\mathbf{u}_{431652} \\
& \equiv \mathbf{u}_{436512}+\mathbf{u}_{436[1,5] 2}+\mathbf{u}_{43[1,6] 52}-\mathbf{u}_{431652}=\mathbf{u}_{436152}-\mathbf{u}_{436152}=0 .
\end{aligned}
$$

In light of the above discussion, it is tempting to conjecture that $\mathfrak{J}_{\lambda}(\mathbf{u})$ is always monomial positive modulo the intersection $\bigcap_{k}\left(I_{\mathrm{A}, k}+I_{\mathrm{st}}\right)$. Unfortunately this turns out to be false, as Proposition 7.4 below shows.

Recall the ideals $I_{\mathrm{L}, k}=J_{\mathrm{L}, k} \cap \mathcal{U}$ from Definition 4.6.

Proposition 7.4 ([6, Corollaries 7.2-7.3]). Let $N=8$. There exists a $\mathrm{D}_{0}$ graph $\Gamma$ on a vertex set $W$ of cardinality 39696 such that

- $\sum_{\mathrm{w} \in W} \mathrm{w} \in\left(\left(I_{\mathrm{plac}}+I_{\mathrm{st}}\right) \cap\left(I_{\mathrm{L}, 3}+I_{\mathrm{st}}\right) \cap\left(I_{\mathrm{L}, 7}+I_{\mathrm{st}}\right)\right)^{\perp} \subset\left(\bigcap_{k}\left(I_{\mathrm{A}, k}+I_{\mathrm{st}}\right)\right)^{\perp} \subset\left(\bigcap_{k} I_{\mathrm{A}, k}\right)^{\perp}$;

- the coefficient of $s_{(2,2,2,2)}(\mathbf{x})$ in $F_{\Gamma}(\mathbf{x})$ is equal to -1 .

Consequently, $\mathfrak{J}_{(2,2,2,2)}(\mathbf{u})$ is not $\mathbb{Q}$-monomial positive modulo $\bigcap_{k}\left(I_{\mathrm{A}, k}+I_{\mathrm{st}}\right)$.

Remark 7.5. It is conceivable that $\mathfrak{J}_{\lambda}(\mathbf{u})$ is $\mathbb{Z}$-monomial positive modulo $\left(I_{\mathrm{A}, k} \cap I_{\mathrm{A}, k+1}\right)$ for any $k$, and that the number of such monomial positive expressions is reasonably small. Then this may be a good route, at least experimentally, towards finding a monomial positive expression for $\mathfrak{J}_{\lambda}(\mathbf{u})$ modulo $I_{\mathrm{A}, k}$ for each particular $k$.

Let $\mathcal{I}_{\lambda}$ be the poset of all ideals $I \supset I_{\mathrm{C}}$, ordered by containment, such that $\mathfrak{J}_{\lambda}(\mathbf{u})$ is $\mathbb{Z}$-monomial positive modulo $I$. What are the minimal elements of $\mathcal{I}_{\lambda}$ ? Are there finitely many such minimal ideals? If $I$ is such a minimal ideal, is there a unique monomial positive expression for $\mathfrak{J}_{\lambda}(\mathbf{u})$ modulo $I$, or can we at least control the number of such expressions? Our investigations suggest that these questions are difficult. One concrete reason is the following surprising corollary of Proposition 7.4 .

Corollary 7.6. There is no unique smallest ideal I containing $I_{\mathrm{C}}$ such that $F_{\gamma}(\mathbf{x})$ is $S$ chur positive for every $\gamma \in \mathcal{U}_{>0}^{*} \cap I^{\perp}$. Similarly, there is no unique smallest ideal I containing $I_{\mathrm{C}}$ such that, for all $\lambda, \overline{\mathfrak{J}}_{\lambda}(\mathbf{u})$ is $\mathbb{Z}$-monomial positive modulo $I$.

Proof. Let $\mathcal{I}$ be the poset of all ideals $I \supset I_{\mathrm{C}}$, ordered by containment, such that $F_{\gamma}(\mathbf{x})$ is Schur positive for every $\gamma \in \mathcal{U}_{\geq 0}^{*} \cap I^{\perp}$. We know that $I_{\mathrm{L}, k} \in \mathcal{I}$ for all $k$ by the Schur positivity of LLT polynomials. We also know that $I_{\text {plac }} \in \mathcal{I}$. If $\mathcal{I}$ has a unique smallest element $J$, then $J \subset \bigcap_{k} I_{\mathrm{L}, k} \cap I_{\text {plac }} \subset\left(I_{\text {plac }}+I_{\text {st }}\right) \cap\left(I_{\mathrm{L}, 3}+I_{\text {st }}\right) \cap\left(I_{\mathrm{L}, 7}+I_{\text {st }}\right)$, hence the latter intersection must lie in $\mathcal{I}$. This however contradicts Proposition 7.4.

The proof of the second statement is similar. We only need the additional fact that $\mathfrak{J}_{\lambda}(\mathbf{u})$ is $\mathbb{Z}$-monomial positive modulo $I_{\mathrm{L}, k}$. This follows from the easy fact that for an ideal $I$ generated by monomials and binomials of the form $\mathbf{u}_{\mathrm{v}}-\mathbf{u}_{\mathrm{w}}, \mathbb{Z}$ - and $\mathbb{Q}$-monomial positivity of $\mathfrak{J}_{\lambda}(\mathbf{u})$ modulo $I$ are equivalent.

One may hope to gain insight into the Schur positivity phenomenon by studying nested sequences of ideals, and identifying the places where a transition from positivity to nonpositivity occurs. Two examples are discussed below. 
Definition 7.7. Let $I_{\mathrm{L}, \leq k}$ denote the ideal in $\mathcal{U}$ generated by the elements

$$
\begin{array}{ll}
u_{a} u_{c}-u_{c} u_{a} & \text { for } c-a>k, \\
u_{b} u_{a} u_{c}+u_{c} u_{a} u_{b}-u_{b} u_{c} u_{a}-u_{a} u_{c} u_{b} & \text { for } c-a \leq k \text { and } a<b<c,
\end{array}
$$

together with all monomials $\mathbf{u}_{\mathrm{w}} \in J_{\mathrm{L}, k}$. Then $I_{\mathrm{L}, k} \supset I_{\mathrm{L}, \leq k} \supset I_{\mathrm{S}}$ and $J_{\mathrm{L}, k} \supset \mathbb{Q}(q) \otimes_{\mathbb{Z}} I_{\mathrm{L}, \leq k}$.

The ideals $I_{\mathrm{L}, \leq k}$ are nested: $I_{\mathrm{L}, \leq k} \subset I_{\mathrm{L}, \leq m}$ for $k \geq m$. Monomial positivity of $\mathfrak{J}_{(2,2,2)}(\mathbf{u})$ fails modulo $I_{\mathrm{L}, \leq 5}$, since it fails modulo $I_{\mathrm{S}}+I_{\mathrm{st}}$ by Corollary 3.9. and $I_{\mathrm{L}, \leq 5} \subset I_{\mathrm{L}, \leq 5}+I_{\mathrm{st}}=$ $I_{\mathrm{S}}+I_{\mathrm{st}}$ for $N=6$. On the other hand, Theorem 4.22 can be strengthened as follows.

Theorem $7.8([5, \S 5.2])$. For any partition $\lambda$, the noncommutative Schur function $\mathfrak{J}_{\lambda}(\mathbf{u})$ is $\mathbb{Z}$-monomial positive modulo $I_{\mathrm{L}, \leq 3}$. A monomial expansion is given by

$$
\mathfrak{J}_{\lambda}(\mathbf{u}) \equiv \sum_{T \in \operatorname{RSST}(\lambda ; N)} \mathbf{u}_{\mathrm{sqread}(T)} \bmod I_{\mathrm{L}, \leq 3} .
$$

As a parallel to our discussion of the ideals $J_{\mathrm{L}, k}$ and $I_{\mathrm{A}, k}$ in Section 4 , it is natural to also study the nested family of ideals $I_{\mathrm{L}, \leq k} \cap I_{\mathrm{A}, k}$ (as $k$ varies). The ideal $I_{\mathrm{L}, \leq k} \cap I_{\mathrm{A}, k}$ contains the elements (2.25)-(2.26), (17.6), and all monomials $\mathbf{u}_{\mathrm{w}} \in J_{\mathrm{L}, k}$. Just as above, monomial positivity of $\mathfrak{J}_{(2,2,2)}(\mathbf{u})$ fails modulo $I_{\mathrm{L}, \leq 5} \cap I_{\mathrm{A}, 5}$. However, we conjecture the following strengthening of Theorem 7.8 (this is a slight variant of a conjecture in [5, §5.2]).

Conjecture 7.9. For any partition $\lambda$, the noncommutative Schur function $\mathfrak{J}_{\lambda}(\mathbf{u})$ is $\mathbb{Z}$ monomial positive modulo $I_{\mathrm{L}, \leq 3} \cap I_{\mathrm{A}, 3}$. A monomial expansion is given by

$$
\mathfrak{J}_{\lambda}(\mathbf{u}) \equiv \sum_{T \in \operatorname{RSST}(\lambda ; N)} \mathbf{u}_{\text {sqread }(T)} \bmod I_{\mathrm{L}, \leq 3} \cap I_{\mathrm{A}, 3} .
$$

Conjecture 7.9 is stronger than the same statement modulo $I_{\mathrm{A}, 3}$, so it would imply that the Assaf symmetric functions of level 3 are Schur positive (cf. Conjecture 4.21).

We conclude this section with a summary of our current knowledge of Schur positivity for symmetric functions associated to switchboards, see Figure 11.

Class of switchboards $\Gamma$

$\mathrm{D}_{0}$ graphs

Switchboards with $\gamma \in\left(\bigcap_{k} I_{\mathrm{A}, k}\right)^{\perp} \quad$ No

Triples switchboards

Assaf switchboards

Switchboards with $\gamma \in\left(I_{\mathrm{L}, \leq 3} \cap I_{\mathrm{A}, 3}\right)^{\perp}$

Switchboards with $\gamma \in I_{\mathrm{L}, \leq 3}^{\perp}$

LLT switchboards

Rotation-free switchboards
All $F_{\Gamma}$ Schur positive? Explanation/proof

No Proposition 3.8

Unknown

Proposition 7.4

Unknown

Problem 6.5

Conjecture 4.21

Unknown

Conjecture 7.9

Yes

Yes

Theorem 7.8 [5]

LLT positivity [27, 15]

Yes
Corollary 5.3 [11]

Figure 11: Schur positivity of symmetric functions $F_{\Gamma}$ for various classes of switchboards $\Gamma$. Here $\gamma$ denotes the sum of the words appearing in $\Gamma$. 


\section{Proof of Theorem 2.10}

We will need the following basic convex geometry lemma (cf., e.g., [30, Theorem 4.1]), which we restate in the form convenient for our purposes.

Lemma 8.1 (Minkowski-Farkas' Lemma). Suppose $\mathbf{a}^{1}, \mathbf{a}^{2}, \ldots, \mathbf{a}^{r} \in \mathbb{Q}^{m}$ and $\mathfrak{J} \in \mathbb{Q}^{m}$. Let $\langle\cdot, \cdot\rangle$ be the standard inner product on $\mathbb{Q}^{m}$. Then the following are equivalent:

- $\mathfrak{J}$ lies in the cone $\mathbb{Q} \geq 0\left\{\mathbf{a}^{1}, \ldots, \mathbf{a}^{r}\right\}$.

- for any $\gamma \in \mathbb{Q}^{m}$ satisfying $\left\langle\mathbf{a}^{i}, \gamma\right\rangle \geq 0$ for all $i$, we have $\langle\mathfrak{J}, \gamma\rangle \geq 0$.

Moreover, in the last line, we can replace $\gamma \in \mathbb{Q}^{m}$ by $\gamma \in \mathbb{Z}^{m}$.

Let $\mathcal{U}_{d}$ denote the degree $d$ homogeneous component of $\mathcal{U}$. For a homogeneous ideal $I$ of $\mathcal{U}$, let $I_{d}=\mathcal{U}_{d} \cap I$ be the degree $d$ homogeneous component of $I$.

Theorem 8.2. Let $I$ be a homogeneous ideal in $\mathcal{U}$. For an element $\mathfrak{J} \in \mathcal{U}_{d}$, the following are equivalent:

(a) $\mathfrak{J}$ is $\mathbb{Q}$-monomial positive modulo $I$;

(b) for any $\gamma \in \mathcal{U}_{\geq 0}^{*} \cap I_{d}^{\perp}$, there holds $\langle\mathfrak{J}, \gamma\rangle \geq 0$.

Proof. Let $\mathbf{u}^{1}, \ldots, \mathbf{u}^{m}$ be the complete list of degree $d$ monomials in $\mathcal{U}_{d}$. Let $\mathbf{g}^{1}, \ldots, \mathbf{g}^{t}$ be a $\mathbb{Q}$-basis of $\mathbb{Q} I_{d}$. We start by observing that $\mathcal{U}_{>0}^{*} \cap I_{d}^{\perp}$ is precisely the set of vectors $\gamma \in \mathcal{U}^{*}$ satisfying $\left\langle\mathbf{u}^{i}, \gamma\right\rangle \geq 0,\left\langle\mathbf{g}^{j}, \gamma\right\rangle \geq 0$, and $\left\langle-\mathbf{g}^{j}, \gamma\right\rangle \geq 0$ for all $i \leq m$ and $j \leq t$. Hence, by Lemma 8.1, condition (b) is equivalent to $\mathfrak{J} \in \mathbb{Q}_{\geq 0}\left\{\mathbf{u}^{1}, \ldots, \mathbf{u}^{m}, \mathbf{g}^{1}, \ldots, \mathbf{g}^{t},-\mathbf{g}^{1}, \ldots,-\mathbf{g}^{t}\right\}$, which is easily seen to be equivalent to condition (a).

We now prove Theorem 2.10, the stronger statement where $\lambda$ is fixed. Let $\lambda$ be a partition of $d$. By Proposition 2.9, the following conditions are equivalent:

(i) for any $\gamma \in \mathcal{U}_{\geq 0}^{*} \cap I^{\perp}$, the coefficient of $s_{\lambda}(\mathbf{x})$ in $F_{\gamma}(\mathbf{x})$ is nonnegative;

$\left(\mathrm{i}^{\prime}\right)$ for any $\gamma \in \mathcal{U}_{\geq 0}^{*} \cap I^{\perp}$, there holds $\left\langle\mathfrak{J}_{\lambda}(\mathbf{u}), \gamma\right\rangle \geq 0$.

Furthermore, by Theorem 8.2 , $\left(\mathrm{i}^{\prime}\right)$ is equivalent to

(ii) $\mathfrak{J}_{\lambda}(\mathbf{u})$ is $\mathbb{Q}$-monomial positive modulo $I$,

establishing the desired equivalence (i) $\Leftrightarrow($ ii).

\section{Proof of Theorem 2.14}

We use the shorthand $[m]=\{1, \ldots, m\}$ throughout. By convention, $[0]=\varnothing$, so for example $e_{k}\left(\mathbf{u}_{[0]}\right)=0$ for $k \neq 0$, and $e_{0}\left(\mathbf{u}_{[0]}\right)=1$. Recall (cf. (2.1)) that $e_{k}\left(\mathbf{u}_{S}\right)$ denotes the noncommutative elementary symmetric function in the variables $\left\{u_{i}\right\}_{i \in S}$.

We will prove Theorem 2.14 by an inductive computation of $\mathfrak{J}_{\lambda}(\mathbf{u})$ which involves peeling off the diagonals of the shape $\lambda$ and incorporating them into a tableau. This requires the following flagged generalization of the noncommutative Schur functions. 
Definition 9.1. For $\alpha=\left(\alpha_{1}, \ldots, \alpha_{l}\right) \in \mathbb{Z}_{\geq 0}^{l}$ and $\mathbf{n}=\left(n_{1}, \ldots, n_{l}\right) \in\{0,1, \ldots, N\}^{l}$, we define the noncommutative column-flagged Schur function $J_{\alpha}(\mathbf{n})=J_{\alpha}(\mathbf{n})(\mathbf{u})$ by

$$
J_{\alpha}(\mathbf{n})=\sum_{\pi \in \mathcal{S}_{l}} \operatorname{sgn}(\pi) e_{\alpha_{1}+\pi(1)-1}\left(\mathbf{u}_{\left[n_{1}\right]}\right) e_{\alpha_{2}+\pi(2)-2}\left(\mathbf{u}_{\left[n_{2}\right]}\right) \cdots e_{\alpha_{l}+\pi(l)-l}\left(\mathbf{u}_{\left[n_{l}\right]}\right) .
$$

These specialize to the noncommutative Schur functions $\mathfrak{J}_{\lambda}(\mathbf{u})$ via $\mathfrak{J}_{\lambda}(\mathbf{u})=J_{\lambda^{\prime}}(N, \ldots, N)$.

Remark 9.2. When the $u_{i}$ commute, $J_{\lambda}(\mathbf{n})$ becomes the column-flagged Schur function $S_{\lambda}^{*}(\mathbf{1}, \mathbf{n})$ studied in [35], where $\mathbf{1}=(1, \ldots, 1) \in \mathbb{Z}^{l}$.

Definition 9.3. Let $\alpha=\left(\alpha_{1}, \ldots, \alpha_{l}\right) \in \mathbb{Z}_{\geq 0}^{l}$ be a composition satisfying $\alpha_{j+1} \leq \alpha_{j}+1$ for all $j$. We denote by $\alpha^{\prime}$ the diagram whose $j$ th column contains the cells in rows $1, \ldots, \alpha_{j}$. Formally, $\alpha^{\prime}=\left\{(i, j) \mid j \in[l], i \in\left[\alpha_{j}\right]\right\} \subset \mathbb{Z}_{\geq 1} \times \mathbb{Z}_{\geq 1}$. Now, for $\mathbf{n}=\left(n_{1}, \ldots, n_{l}\right) \in \mathbb{Z}_{\geq 0}^{l}$, let $\operatorname{SSYT}\left(\alpha^{\prime}\right)^{\mathbf{n}}$ denote the set of fillings $T: \mathbb{Z}_{\geq 1} \times \mathbb{Z}_{\geq 1} \rightarrow[N]$ of the diagram $\alpha^{\prime}$ such that

- the entries weakly increase across the rows and strictly increase down the columns;

- the entries in column $j$ lie in $\left[n_{j}\right]$;

- if $\alpha_{j}<\alpha_{j+1}$, then $T\left(\alpha_{j+1}, j+1\right)>n_{j}$.

The diagonal reading word of $T \in \operatorname{SSYT}\left(\alpha^{\prime}\right)^{\mathbf{n}}$, denoted diagread $(T)$, is obtained by concatenating the diagonals of $T$ (reading each diagonal in the southeast direction), starting with the southwesternmost diagonal of $\alpha^{\prime}$. For example,

$$
\operatorname{diagread}\left(\begin{array}{l|l|l|l}
\hline 1 & 1 & 2 & 2 \\
\hline 2 & 2 & 3 & \\
\cline { 1 - 2 } & \multicolumn{1}{|c}{} & 7 &
\end{array}\right)=21271322
$$

Theorem 9.4 below is a generalization of Theorem 2.14. To obtain Theorem 2.14 from it, set $n_{1}=\cdots=n_{l}=N$ and $\alpha=\lambda^{\prime}$.

Theorem 9.4. Let $\alpha=\left(\alpha_{1}, \ldots, \alpha_{l}\right) \in \mathbb{Z}_{\geq 0}^{l}$ satisfy $\alpha_{j+1} \leq \alpha_{j}+1$ for all $j$, and let $0 \leq n_{1} \leq \cdots \leq n_{l} \leq N$. Then

$$
J_{\alpha}(\mathbf{n})=J_{\alpha}\left(n_{1}, \ldots, n_{l}\right) \equiv \sum_{T \in \operatorname{SSYT}\left(\alpha^{\prime}\right)^{\mathbf{n}}} \mathbf{u}_{\operatorname{diagread}(T)} \bmod I_{\mathrm{B}}
$$

We will prove Theorem 9.4 by establishing its more technical variant Theorem 9.6 , see below. To state the latter, we will need the following definition.

Definition 9.5. The augmented noncommutative column-flagged Schur function labeled by a weak composition $\alpha=\left(\alpha_{1}, \ldots, \alpha_{l}\right) \in \mathbb{Z}_{\geq 0}^{l}$, an $l$-tuple $\mathbf{n}=\left(n_{1}, \ldots, n_{l}\right) \in\{0,1, \ldots, N\}^{l}$, and words $\mathrm{w}^{1}, \ldots, \mathrm{w}^{l-1} \in \mathcal{U}^{*}$ is defined by

$$
\begin{aligned}
& J_{\alpha}\left(n_{1}: \mathrm{w}^{1}: n_{2}: \mathrm{w}^{2}: \cdots: \mathrm{w}^{l-1}: n_{l}\right) \\
& =\sum_{\pi \in \mathcal{S}_{l}} \operatorname{sgn}(\pi) e_{\alpha_{1}+\pi(1)-1}\left(\mathbf{u}_{\left[n_{1}\right]}\right) \mathbf{u}_{\mathrm{w}^{1}} e_{\alpha_{2}+\pi(2)-2}\left(\mathbf{u}_{\left[n_{2}\right]}\right) \mathbf{u}_{\mathrm{w}^{2}} \cdots \mathbf{u}_{\mathrm{w}^{l-1}} e_{\alpha_{l}+\pi(l)-l}\left(\mathbf{u}_{\left[n_{l}\right]}\right) .
\end{aligned}
$$

We omit $\mathbf{w}^{j}$ from the notation in (9.3) if the word $\mathbf{w}^{j}$ is empty.

The peeling index of $\alpha=\left(\alpha_{1}, \ldots, \alpha_{l}\right) \in \mathbb{Z}_{\geq 0}^{l}$ is the smallest $j$ such that $\alpha_{j} \geq \alpha_{j+1}$. Here $\alpha_{l+1}=0$ by convention. 
For a word $\mathrm{w}$ and a filling $T$ of a diagram $\alpha^{\prime}$ (as in Definition 9.3), define the word $\operatorname{diagread}(\mathbf{w} ; T)$ as follows: if $\alpha_{1}=\alpha_{2}=0, \operatorname{set} \operatorname{diagread}(\mathbf{w} ; T)=\mathrm{w} \operatorname{diagread}(T)$. Otherwise, $\operatorname{diagread}(\mathrm{w} ; T)=\mathrm{d}^{1} \mathrm{wd}^{2} \ldots \mathrm{d}^{\mathrm{t}}$, where $\mathrm{d}^{1}, \mathrm{~d}^{2}, \ldots, \mathrm{d}^{\mathrm{t}}$ are the strictly increasing words corresponding to the (nonempty) diagonals of $T$, numbered starting with the southwesternmost diagonal. (Hence diagread $(\mathbf{w} ; T)=\operatorname{diagread}(T)$ when $w$ is empty.)

Theorem 9.6. Let $\alpha=\left(\alpha_{1}, \ldots, \alpha_{l}\right) \in \mathbb{Z}_{>0}^{l}$ be such that $\alpha_{i+1} \leq \alpha_{i}+1$ for all $i$. Let $j$ be the peeling index of $\alpha$. If $0 \leq n_{1} \leq n_{2} \leq \cdots \leq n_{l}$, and $\mathrm{w}$ is a strictly increasing word in letters $>n_{j}$, then

$$
J_{\alpha}\left(n_{1}, \ldots, n_{j-1}, n_{j}: \mathrm{w}: n_{j+1}, \ldots\right) \equiv \sum_{T \in \operatorname{SSYT}\left(\alpha^{\prime}\right)^{\mathbf{n}}} \mathbf{u}_{\operatorname{diagread}(\mathrm{w} ; T)} \bmod I_{\mathrm{B}}
$$

Proof. Write $J$ for $J_{\alpha}\left(n_{1}, \ldots, n_{j}: \mathrm{w}: n_{j+1}, \ldots\right)$. The proof is by induction on $l$ and the $n_{i}$.

We first address the base cases $\left(\alpha_{1}=0, j=1\right)$ and $\left(n_{1}=0, j=1\right)$. Suppose $\alpha_{1}=0$ and $j=1$. Since $\alpha_{i+1} \leq \alpha_{i}+1$ for all $i$ and $\alpha_{1}=\alpha_{2}=0, J_{\alpha}\left(n_{1}, \ldots, n_{l}\right)$ is a noncommutative version of the determinant of a matrix whose first column, read downwards, is a 1 followed by 0 's. Then by Definition 9.5 and induction,

$$
J=\mathbf{u}_{\mathrm{w}} J_{\hat{\alpha}}(\hat{\mathbf{n}}) \equiv \mathbf{u}_{\mathrm{w}} \sum_{T \in \operatorname{SSYT}\left(\hat{\alpha}^{\prime}\right)^{\hat{\mathbf{n}}}} \mathbf{u}_{\operatorname{diagread}(T)}=\sum_{T \in \operatorname{SSYT}\left(\alpha^{\prime}\right)^{\mathbf{n}}} \mathbf{u}_{\operatorname{diagread}(\mathrm{w} ; T)} \bmod I_{\mathrm{B}},
$$

where $\hat{\alpha}=\left(\alpha_{2}, \ldots, \alpha_{l}\right)$ and $\hat{\mathbf{n}}=\left(n_{2}, \ldots, n_{l}\right)$. Now suppose $n_{1}=0$ and $j=1$. By the previous case, we may assume $\alpha_{1}>0$. Then $J_{\alpha}\left(n_{1}, \ldots, n_{l}\right)$ is a noncommutative version of the determinant of a matrix whose first row is 0 , hence $J=0$. Since $n_{1}=0$ implies that $\operatorname{SSYT}\left(\alpha^{\prime}\right)^{\mathbf{n}}$ is empty, the right-hand side of (9.5) is also 0.

The argument for the induction step requires the following three lemmas.

Lemma 9.7. If $\alpha_{j+1}=\alpha_{j}+1$ and $n_{j+1}=n_{j}$, then $J_{\alpha}(\mathbf{n}) \equiv 0 \bmod I_{\mathrm{B}}$. More generally, this holds in the augmented case provided $\mathrm{w}^{j}$ is empty.

Proof. This follows from the fact that the noncommutative elementary symmetric functions commute modulo $I_{\mathrm{B}}$.

Lemma 9.8. If the word $\mathrm{v}=\mathrm{v}_{1} \cdots \mathrm{v}_{s}$ is strictly decreasing (i.e., $v_{1}>\cdots>v_{s}$ ) and $\mathrm{w}=$ $\mathrm{w}_{1} \cdots \mathrm{w}_{t}$ is strictly increasing, and moreover $v_{1}<b<w_{1}$, then $u_{b} \mathbf{u}_{\mathrm{v}} \mathbf{u}_{\mathrm{w}} \equiv u_{b} \mathbf{u}_{\mathrm{w}} \mathbf{u}_{\mathrm{v}} \bmod I_{\mathrm{B}}$.

Proof. The case $t=1$ follows immediately from $s$ applications of the plactic relation with three distinct letters. Applying this special case $t$ times gives the result: $u_{b} \mathbf{u}_{\mathrm{v}} u_{w_{1}} \cdots u_{w_{t}} \equiv$ $u_{b} u_{w_{1}} \mathbf{u}_{\mathrm{v}} u_{w_{2}} \cdots u_{w_{t}} \equiv u_{b} u_{w_{1}} u_{w_{2}} \mathbf{u}_{\mathrm{v}} u_{w_{3}} \cdots u_{w_{t}} \equiv \cdots \equiv u_{b} \mathbf{u}_{\mathrm{w}} \mathbf{u}_{\mathrm{v}} \bmod I_{\mathrm{B}}$.

Lemma 9.9. Let $m \in \mathbb{Z}_{>0}$ and $k \in \mathbb{Z}$. Then

$$
e_{k}\left(\mathbf{u}_{[m]}\right)=u_{m} e_{k-1}\left(\mathbf{u}_{[m-1]}\right)+e_{k}\left(\mathbf{u}_{[m-1]}\right) .
$$

Proof. This is immediate from the definition of $e_{k}\left(\mathbf{u}_{[m]}\right)$.

For an index $j \in[l]$, we will apply (9.7) to $J_{\alpha}\left(n_{1}, \ldots, n_{l}\right)$ and its variants by expanding $e_{\alpha_{j}+\pi(j)-j}\left(\mathbf{u}_{\left[n_{j}\right]}\right)$ in (9.1) using (9.7) (so that (9.7) is applied once to each of the $l$ ! terms in the sum in (9.1)). We refer to this as a $j$-expansion of $J_{\alpha}\left(n_{1}, \ldots, n_{l}\right)$ using (9.7). 
Set $\alpha_{-}=\left(\alpha_{1}, \ldots, \alpha_{j-1}, \alpha_{j}-1, \alpha_{j+1}, \ldots\right)$ and $\mathbf{n}_{-}=\left(n_{1}, \ldots, n_{j-1}, n_{j}-1, n_{j+1}, \ldots\right)$. By the base cases above, we may assume that either $\left(\alpha_{1}>0\right.$ and $n_{1}>0$ and $\left.j=1\right)$ or $j>1$. We first handle the former case. A 1-expansion of $J$ using (9.7) yields

$$
\begin{aligned}
J & =u_{n_{1}} J_{\alpha_{-}}\left(n_{1}-1: \mathrm{w}: n_{2}, \ldots, n_{l}\right)+J_{\alpha}\left(n_{1}-1: \mathrm{w}: n_{2}, \ldots, n_{l}\right) \\
& \equiv u_{n_{1}} \mathbf{u}_{\mathrm{w}} J_{\alpha_{-}}\left(n_{1}-1, n_{2}, \ldots, n_{l}\right)+J_{\alpha}\left(n_{1}-1: \mathrm{w}: n_{2}, \ldots, n_{l}\right) \\
& \equiv u_{n_{1}} \mathbf{u}_{\mathrm{w}} \sum_{T \in \operatorname{SSYT}\left(\alpha_{-}^{\prime}\right)^{\mathbf{n}_{-}}} \mathbf{u}_{\operatorname{diagread}(T)}+\sum_{T \in \operatorname{SSYT}\left(\alpha^{\prime}\right)^{\mathbf{n}_{-}}} \mathbf{u}_{\operatorname{diagread}(\mathrm{w} ; T)} \bmod I_{\mathrm{B}} .
\end{aligned}
$$

The first congruence is by Lemma 9.8 and the second is by induction. Now (9.9) is equal to the right-hand side of (9.5) because (9.9) is the result of partitioning the set of tableaux appearing in (9.5) into two, depending on whether or not $T\left(\alpha_{j}, j\right)$ is equal to or less than $n_{j}$. Note that if $\alpha_{j}=\alpha_{j+1}$, then for $T \in \operatorname{SSYT}\left(\alpha^{\prime}\right)^{\mathbf{n}}$ with $T\left(\alpha_{j}, j\right)=n_{j}$, the condition $T\left(\alpha_{j}, j\right) \leq T\left(\alpha_{j+1}, j+1\right)$ (which holds since the rows of $T$ are weakly increasing) corresponds to the condition $n_{j}-1=\left(n_{-}\right)_{j}<T^{\prime}\left(\left(\alpha_{-}\right)_{j+1}, j+1\right)=T^{\prime}\left(\alpha_{j+1}, j+1\right)$ required of any $T^{\prime} \in \operatorname{SSYT}\left(\alpha_{-}^{\prime}\right)^{\mathbf{n}_{-}}$.

Now let $j>1$. By definition of the peeling index, $\alpha_{j}=\alpha_{j-1}+1$ and $\alpha_{j} \geq \alpha_{j+1}$. If $n_{j-1}=n_{j}$, then $J \equiv 0 \bmod I_{\mathrm{B}}$ by Lemma 9.7, in this case $\operatorname{SSYT}\left(\alpha^{\prime}\right)^{\mathbf{n}}$ is empty, so the right-hand side of (9.5) is also 0 . So we may assume that $n_{j-1}<n_{j}$. Now by a $j$-expansion of $J$ using (9.7), we have

$$
\begin{aligned}
J= & J_{\alpha_{-}}\left(n_{1}, \ldots, n_{j-1}: \mathrm{n}_{\mathrm{j}}: n_{j}-1: \mathrm{w}: n_{j+1}, \ldots\right) \\
& +J_{\alpha}\left(n_{1}, \ldots, n_{j-1}, n_{j}-1: \mathrm{w}: n_{j+1}, \ldots\right) \\
\equiv & J_{\alpha_{-}}\left(n_{1}, \ldots, n_{j-1}: \mathrm{n}_{\mathrm{j}} \mathrm{w}: n_{j}-1, n_{j+1}, \ldots\right) \\
& +J_{\alpha}\left(n_{1}, \ldots, n_{j-1}, n_{j}-1: \mathrm{w}: n_{j+1}, \ldots\right) \\
\equiv & \sum_{T \in \operatorname{SSYT}\left(\alpha_{-}^{\prime}\right)^{\mathbf{n}_{-}}} \mathbf{u}_{\text {diagread }\left(\mathrm{n}_{\mathrm{j}} \mathrm{w} ; T\right)}+\sum_{T \in \operatorname{SSYT}\left(\alpha^{\prime}\right)^{\mathbf{n}_{-}}} \mathbf{u}_{\operatorname{diagread}(\mathrm{w} ; T)} \bmod I_{\mathrm{B}} .
\end{aligned}
$$

The first congruence is by Lemma 9.8. We now justify the second congruence. Since the tuple $\mathbf{n}_{-}$is weakly increasing, $j-1$ is the peeling index of $\alpha_{-}$, and $\mathbf{n}_{j} \mathbf{w}$ is a strictly increasing word in letters $>n_{j-1}$, the summand (9.10) satisfies the conditions of Theorem 9.6, hence by induction, it is congruent $\left(\bmod I_{\mathrm{B}}\right)$ to the first sum in $(9.12)$. Since the tuple $\mathbf{n}_{-}$is weakly increasing, the summand (9.11) is congruent to the second sum in (9.12) by induction. Finally, (9.12) is equal to the right-hand side of (9.5) by the same argument given in the $j=1$ case.

We conclude by reconciling the difference between the reading words in Theorems 5.1 and 9.4 .

Proposition 9.10. For any $S S Y T T, \mathbf{u}_{\operatorname{colword}(T)} \equiv \mathbf{u}_{\operatorname{diagread}(T)} \bmod I_{\mathrm{B}}$. Hence either the diagonal reading word or the column reading word can be used in Theorems 5.1 and 9.4.

Proof. The proof is by induction on the number of cells of $T$. Let $\mathrm{d}^{1}, \mathrm{~d}^{2}, \ldots, \mathrm{d}^{\mathrm{t}}$ be the strictly increasing words corresponding to the diagonals of $T$, so that diagread $(T)=$ $\mathrm{d}^{1} \mathrm{~d}^{2} \cdots \mathrm{d}^{\mathrm{t}}$. Let $r$ be the number of rows of $T$. For each $i=1,2, \ldots, r$, set $\mathrm{d}^{\mathrm{i}}=\mathrm{c}_{\mathrm{i}} \hat{\mathrm{d}}^{\mathrm{i}}$ so that 
$c_{i}$ is the first letter of $d^{i}$ and $\hat{d}^{i}$ is the remainder of the word $d^{i}$. The key computation is

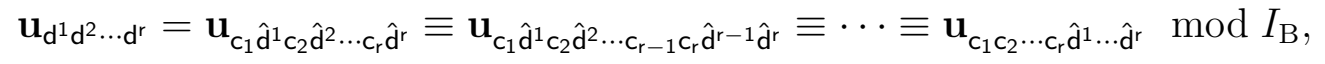

where each congruence is by Lemma 9.8 (whose hypotheses are satisfied since the $\mathrm{d}^{\mathrm{i}}$ are strictly increasing and $c_{1}>c_{2}>\cdots>c_{r}$ ). Now let $T^{\prime}$ be the tableau obtained from $T$ by removing the first column. Note that $c_{1} \cdots c_{r}$ is the first column of $T$ read bottom to top, and $\hat{d}^{1} \ldots \hat{d}^{r} d^{r+1} \ldots d^{t}$ is the diagonal reading word of $T^{\prime}$. Hence by (9.13) and induction, we have $\mathbf{u}_{\text {diagread }(T)} \equiv \mathbf{u}_{\mathrm{c}_{1} \cdots \mathrm{c}_{\mathrm{r}}} \mathbf{u}_{\text {diagread }\left(T^{\prime}\right)} \equiv \mathbf{u}_{\mathrm{c}_{1} \cdots \mathrm{c}_{\mathrm{r}}} \mathbf{u}_{\text {colword }\left(T^{\prime}\right)} \equiv \mathbf{u}_{\operatorname{colword}(T)} \bmod I_{\mathrm{B}}$.

\section{REFERENCES}

[1] S. H. Assaf, Dual equivalence graphs and a combinatorial proof of LLT and Macdonald positivity, arXiv:1005.3759v5, October 2013.

[2] S. H. Assaf, Dual equivalence and Schur positivity, preprint, March 2014. The content of this paper has been included in 3 .

[3] S. H. Assaf, Dual equivalence graphs I: A new paradigm for Schur positivity, Forum Math. Sigma 3 (2015), e12, 33 pp.

[4] J. Blasiak and R. I. Liu, Kronecker coefficients and noncommutative super Schur functions, arXiv: 1510.00644 .

[5] J. Blasiak, Haglund's conjecture on 3-column Macdonald polynomials, Math. Z. 283 (2016), 601-628.

[6] J. Blasiak, What makes a $\mathrm{D}_{0}$ graph Schur positive? J. Algebraic Combin. (2016), 1-51.

[7] W. Bosma, J. Cannon, and C. Playoust, The Magma algebra system. I. The user language, J. Symbolic Comput. 24 (1997), 235-265.

[8] C. Carré and B. Leclerc, Splitting the square of a Schur function into its symmetric and antisymmetric parts, J. Algebraic Combin. 4 (1995), 201-231.

[9] P. Edelman and C. Greene, Balanced tableaux, Adv. in Math. 63 (1987), 42-99.

[10] S. Fishel, Statistics for special q,t-Kostka polynomials, Proc. Amer. Math. Soc. 123 (1995), 29612969.

[11] S. Fomin and C. Greene, Noncommutative Schur functions and their applications, Discrete Math. 193 (1998), 179-200.

[12] S. Fomin and R. P. Stanley, Schubert polynomials and the nil-Coxeter algebra, Adv. Math. 103 (1994), 196-207.

[13] A. M. Garsia and M. Haiman, A graded representation model for Macdonald's polynomials, Proc. Nat. Acad. Sci. U.S.A. 90 (1993), 3607-3610.

[14] I. M. Gessel, Multipartite $P$-partitions and inner products of skew Schur functions, Contemp. Math., vol. 34, 289-317, Amer. Math. Soc., Providence, RI, 1984.

[15] I. Grojnowski and M. Haiman, Affine Hecke algebras and positivity of LLT and Macdonald polynomials, preprint, 2007.

[16] J. Haglund, M. Haiman, and N. Loehr, A combinatorial formula for Macdonald polynomials, J. Amer. Math. Soc. 18 (2005), 735-761.

[17] J. Haglund, M. Haiman, N. Loehr, J. B. Remmel, and A. Ulyanov, A combinatorial formula for the character of the diagonal coinvariants, Duke Math. J. 126 (2005), 195-232.

[18] M. Haiman, Combinatorics, symmetric functions, and Hilbert schemes, Current developments in mathematics, 2002, 39-111, Int. Press, Somerville, MA, 2003.

[19] M. Kashiwara and T. Tanisaki, Parabolic Kazhdan-Lusztig Polynomials and Schubert Varieties, J. Algebra 249 (2002), 306-325.

[20] D. Kazhdan and G. Lusztig, Representations of Coxeter groups and Hecke algebras, Invent. Math. 53 (1979), 165-184.

[21] A. N. Kirillov, Notes on Schubert, Grothendieck and key polynomials, SIGMA 12 (2016), 57 pp.

[22] T. Lam, Ribbon Schur operators, European J. Combin. 29 (2008), 343-359. 
[23] L. Lapointe and J. Morse, Tableaux statistics for two part Macdonald polynomials, Algebraic combinatorics and quantum groups, 61-84, World Sci. Publ., River Edge, NJ, 2003.

[24] A. Lascoux, B. Leclerc, and J.-Y. Thibon, Ribbon tableaux, Hall-Littlewood functions, quantum affine algebras, and unipotent varieties, J. Math. Phys. 38 (1997), 1041-1068.

[25] A. Lascoux and M.-P. Schützenberger, Le monoïde plaxique, Noncommutative structures in algebra and geometric combinatorics (Naples, 1978), 129-156, Quad. "Ricerca Sci.", 109, CNR, Rome, 1981.

[26] A. Lascoux and M.-P.-Schützenberger, Structure de Hopf de l'anneau de cohomologie et de l'anneau de Grothendieck d'une variété de drapeaux, C. R. Acad. Sci. Paris Sér. I Math. 295 (1982), 629-633.

[27] B. Leclerc and J.-Y. Thibon, Littlewood-Richardson coefficients and Kazhdan-Lusztig polynomials, Combinatorial methods in representation theory (Kyoto, 1998), 155-220, Adv. Stud. Pure Math., 28, Kinokuniya, Tokyo, 2000.

[28] J.-C. Novelli and A. Schilling, The forgotten monoid, Combinatorial representation theory and related topics, 71-83, RIMS Kôkyûroku Bessatsu, B8, Res. Inst. Math. Sci. (RIMS), Kyoto, 2008.

[29] A. Roberts, Dual equivalence graphs revisited and the explicit Schur expansion of a family of LLT polynomials, J. Algebraic Combin. 39 (2014), 389-428.

[30] J. C. Rosales and P. A. García-Sánchez, Finitely generated commutative monoids, Nova Science Publishers, Inc., Commack, NY, 1999.

[31] M.-P. Schützenberger, La correspondance de Robinson, Combinatoire et représentation du groupe symétrique, 59-113, Lecture Notes in Math., 579, Springer, Berlin, 1977.

[32] R. P. Stanley, Enumerative combinatorics, vol. 2, Cambridge University Press, Cambridge, 1999.

[33] J. R. Stembridge, Admissible $W$-graphs, Represent. Theory 12 (2008), 346-368.

[34] M. A. A. van Leeuwen, Some bijective correspondences involving domino tableaux, Electron. J. Combin. 7 (2000), Research Paper 35, 25 pp.

[35] M. L. Wachs, Flagged Schur functions, Schubert polynomials, and symmetrizing operators, J. Combin. Theory Ser. A 40 (1985), 276-289.

[36] M. Zabrocki, Positivity for special cases of $(q, t)$-Kostka coefficients and standard tableaux statistics, Electron. J. Combin. 6 (1999), Research Paper 41, 36 pp.

Department of Mathematics, Drexel University, Philadelphia, PA 19104

E-mail address: jblasiak@gmail.com

Department of Mathematics, University of Michigan, Ann Arbor, Mi 48109

E-mail address: fomin@umich.edu 